\title{
Universiteit
}

Leiden

The Netherlands

\section{Chromosome organization in bacteria: mechanistic insights into genome structure and function}

Dame, R.T.; Rashid F.Z.M.; Grainger, D.C.

\section{Citation}

Dame, R. T., \& Grainger, D. C. (2019). Chromosome organization in bacteria: mechanistic insights into genome structure and function. Nature Reviews Genetics, 21, 227-242. doi:10.1038/s41576-019-0185-4

Version:

Publisher's Version

License:

Licensed under Article 25fa Copyright Act/Law (Amendment Taverne)

Downloaded from: https://hdl.handle.net/1887/3248592

Note: To cite this publication please use the final published version (if applicable). 


\title{
Chromosome organization in bacteria: mechanistic insights into genome structure and function
}

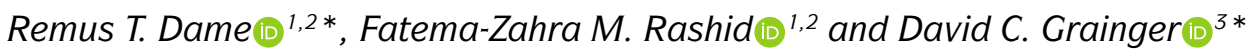 \\ Abstract | Bacterial chromosomes are folded to compact DNA and facilitate cellular processes. \\ Studying model bacteria has revealed aspects of chromosome folding that are applicable to many \\ species. Primarily controlled by nucleoid-associated proteins, chromosome folding is hierarchical, \\ from large-scale macrodomains to smaller-scale structures that influence DNA transactions, \\ including replication and transcription. Here we review the environmentally regulated, \\ architectural and regulatory roles of nucleoid-associated proteins and the implications for \\ bacterial cell biology. We also highlight similarities and differences in the chromosome folding \\ mechanisms of bacteria and eukaryotes.
}

Chromosome

An essential molecule

containing some or all of the genes required by an organism to survive and reproduce. Whereas chromosomes are made of DNA, not all DNA is chromosomal. Extrachromosomal DNA molecules such as plasmids also encode genes, although these genes are not absolutely required for an organism's survival and reproduction.

Chromatin A compact macromolecular complex of DNA and structuring proteins.
'Leiden Institute of Chemistry, Leiden University, Leiden, Netherlands.

${ }^{2}$ Centre for Microbial Cell Biology, Leiden University, Leiden, Netherlands.

${ }^{3}$ Institute of Microbiology and Infection, School of Biosciences, University of Birmingham, Birmingham, UK

*e-mail: rtdame@ chem.leidenuniv.nl; d.grainger@bham.ac.uk https://doi.org/10.1038/ s41576-019-0185-4
In all organisms, DNA is folded to fit inside the cell or its compartments. This is necessary because an organism's chromosome exceeds the cell's length by several orders of magnitude. Since the 1950s, 'spreads' of liberated intracellular macromolecules, visualized by electron microscopy, have demonstrated the difficulty of this task. Genetic material readily spills out of lysed cells or nuclei to fill a volume many times larger than originally occupied $^{1}$. The precise mechanisms of DNA folding were first understood for eukaryotes. Hence, the basic structural units of eukaryotic folded DNA (nucleosomes; FIG. 1a) were identified as 'beads on a string" ${ }^{2}$. The discovery of higher-order structures (chromatin) was facilitated by the large size of eukaryotic cells, which makes them more amenable to light microscopy. Indeed, the basic dynamics of eukaryotic chromosomes during cell division were evident even before the genetic code was understood ${ }^{3}$. It has taken much longer to understand chromosome organization in bacteria. Repeating structural units have never been identified, and early visualizations showed little more than a tangled mess ${ }^{1}$. In retrospect, this is unsurprising. Bacteria lack most DNA-folding factors present in eukaryotes, so few cues can be taken. Furthermore, bacterial nucleoids undergo large changes in organization at different growth phases.

When we previously reviewed this topic in 2011 $\left(\mathrm{REF}^{4}\right)$, evidence was emerging that bacterial chromosomes are not merely unstructured bodies of DNA but instead fold into independent domains finely structured at the nanoscale. Advances in microscopy, structural biology and genome-scale approaches (BOX 1) have revealed many of the underlying molecular mechanisms. In this Review, we discuss these mechanisms and their impact on wider cell biology. Beginning at the level of individual
DNA-folding proteins, we explain how DNA in bacteria is folded into myriad structures by looping, bending and twisting of the DNA. Subsequently, we explain how these DNA contortions can influence not only nucleic acid compaction but also gene expression and DNA replication. On a whole-chromosome scale, we describe the characteristics of individual domains and discuss the possibility that this organization concept is conserved across bacterial species. Throughout, we highlight similarities and differences in the DNA-folding mechanisms used by bacteria and eukaryotes.

\section{The nucleoid-associated proteins}

Unlike eukaryotes, chromosomes of bacteria are not usually folded into regularly repeating structural units (FIG. 1a). Instead, the chromosome is folded into a range of different conformations by nucleoid-associated proteins (NAPs) (FIG. $1 \mathrm{~b}-\mathrm{e}$ ). These are described further in the following sections.

Loop and filament formation by $\mathbf{H}$-NS. The histonelike nucleoid structuring protein (H-NS) is a small (137 amino acids in Escherichia coli) polypeptide that binds the DNA minor groove using a carboxy-terminal arginine hook motif ${ }^{5}$. This is favoured for DNA with elevated AT content containing a TpA dinucleotide or

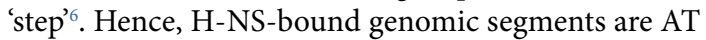
rich and have often been acquired by horizontal gene transfer ${ }^{7-11}$. The amino-terminal domain of H-NS contains two sites that facilitate 'daisy chaining' of H-NS via head-to-head and tail-to-tail contacts ${ }^{12}$ (FIG. 1 b). This drives the formation of lateral nucleoprotein filaments (FIG. 1b, top) or loops between DNA segments bridged by $\mathrm{H}-\mathrm{NS}^{13,14}$ (FIG. 1 b, bottom). 
a

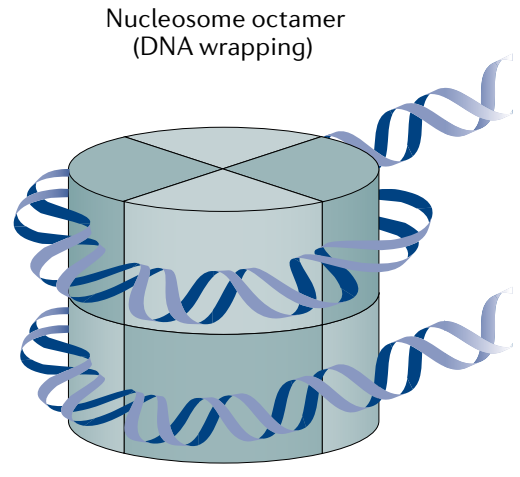

b

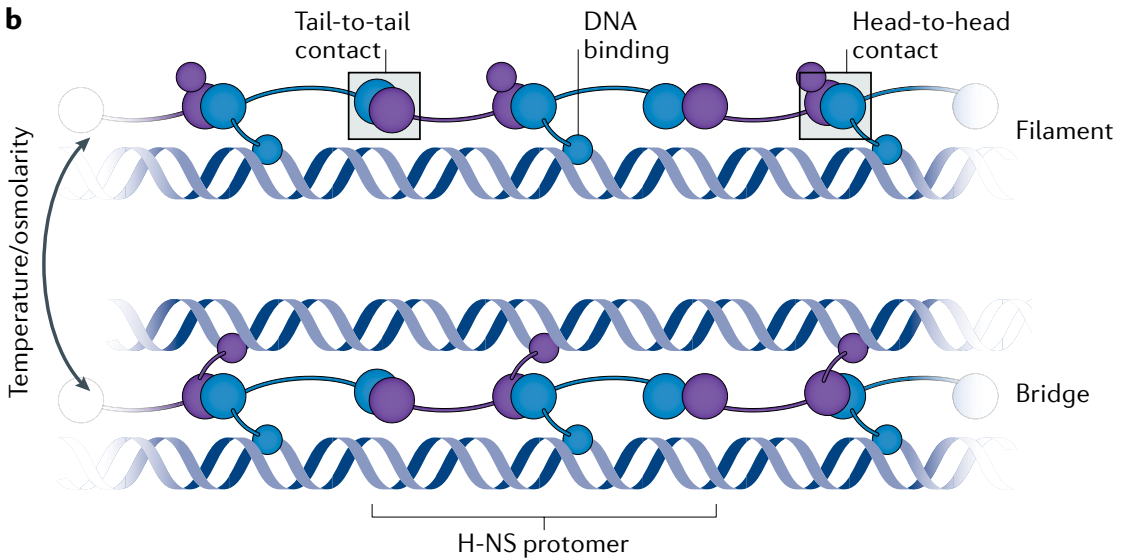

c

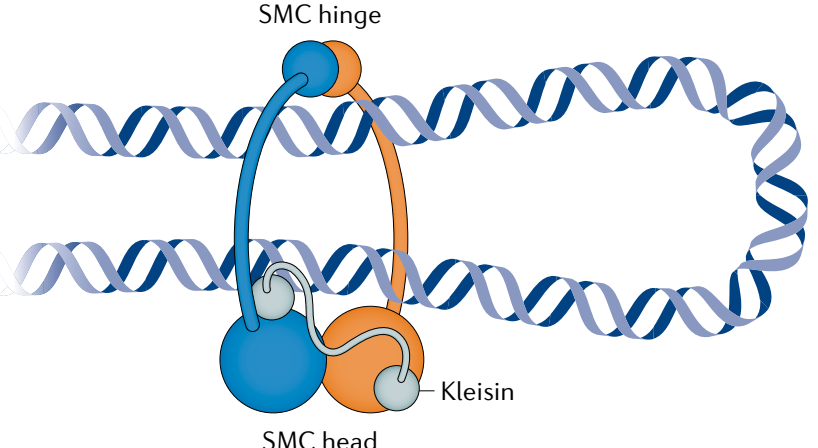

d

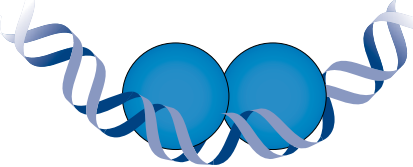

Fis (DNA bending) e

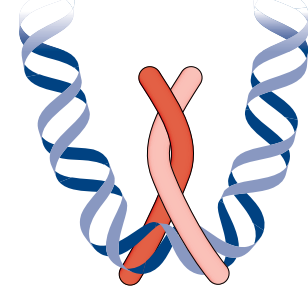

IHF or HU (DNA bending)

Fig. 1 | DNA is locally folded by nucleoid-associated proteins in bacteria, histones in eukaryotes and the evolutionarily conserved SMC complex. a Eukaryotic chromosomes are folded into regularly repeating structural units known as nucleosomes. Typically, a nucleosome consists of $147 \mathrm{bp}$ of DNA wrapped around an octameric histone core. b | The 'daisy chaining' of histone-like nucleoid structuring protein (H-NS) along the DNA by head-to-head and tail-to-tail contacts forms H-NS-DNA filaments (top) and DNA-H-NS-DNA bridges (bottom), the latter of which results in the formation of DNA loops ${ }^{12-14}$. In vitro studies indicate that the switch between the two modes of DNA binding by H-NS is mediated by changes in temperature and osmolarity ${ }^{63,123-126} . \mathbf{c}$ | Structural maintenance of chromosomes (SMC) complexes are DNA-looping proteins comprising a pair of SMC monomers, kleisin and the kite (kleisin-interacting winged-helix tandem elements) or hawk (HEAT repeat subunits containing proteins associated with kleisins) accessory/regulatory proteins. Each SMC monomer consists of a 'hinge' dimerization domain, an ATPase 'head' domain and an antiparallel coiled-coil 'arm' extending between the hinge and head domains. SMC complexes form DNA loops either by embracing a pair of DNA segments in a single ring or by the dimerization of two rings that each trap a DNA segment ${ }^{19-24}$ (also see FIG. 2). d | Factor for inversion stimulation (Fis) binds its target sequences as a dimer and induces a 50-90 bend in the DNA ${ }^{\circ 7,48}$. e Integration host factor (IHF) and heat-stable protein from E. coli strain U93 (HU) also function as DNA-bending proteins. IHF generates sharp $160^{\circ}$ hairpin bends in the DNA, whereas $\mathrm{HU}$ functions as a flexible hinge ${ }^{51}$ - it bends DNA less sharply but over a range of different angles ${ }^{55,56}$.

Nucleoids

Structures found in prokaryotic cells that contain chromosomes, bound proteins and other associated molecules (for example, RNAs). Nucleoids are functionally similar to the nuclei of eukaryotic cells but are not enclosed within a membrane. Nucleoids can be found in eukaryotic organelles believed to be bacterial in origin.

Genome

The complete set of genes encoded by the DNA content of a given organism. The genome includes genes encoded by chromosomal and extrachromosomal DNA, and intervening non-coding regions.
Proteins functionally similar to H-NS are found in diverse bacteria. Often these have arisen via convergent evolution: that is, the independent evolution of the same function. For example, in Burkholderia spp. and Mycobacterium tuberculosis, the functional equivalents of E. coli $\mathrm{H}-\mathrm{NS}$ are Bv3f and Lsr2, respectively. The proteins share structural similarity only in the arginine hook motif responsible for DNA binding ${ }^{6,15}$. Furthermore, Bacillus subtilis Rok shares no structural similarity with H-NS, Bv3f or Lsr2 yet fulfils the same physiological role ${ }^{16}$, by binding AT-rich DNA and having a strong preference for sequences containing a TpA step ${ }^{17}$. The interaction is mediated by lysine side chains in a winged helix that make contacts with the DNA backbone ${ }^{17}$. The MvaT protein of Pseudomonas species uses lysine residues in an AT-pincer motif to make similar contacts ${ }^{18}$.
DNA looping by SMC proteins. Structural maintenance of chromosomes (SMC) complexes are tripartite rings composed of a pair of SMC monomers, kleisin and the accessory/regulatory kite (kleisin-interacting wingedhelix tandem elements) or hawk (HEAT repeat subunits containing proteins associated with kleisins) proteins $^{19-22}$. Each SMC monomer consists of a 'hinge' dimerization domain involved in the formation of a V-shaped SMC dimer, an ATPase 'head' domain and an antiparallel coiled-coil 'arm' extending between the hinge and head domains. The SMC dimer is bound to a kleisin complex to form a ring that captures DNA ${ }^{19,21,23,24}$ and, by encompassing two DNA segments, forms a loop $^{25}$ (FIG. 1 C, FIG. 2, top). Such a loop may also form by the dimerization of SMC dimers that each embrace a DNA segment ${ }^{26}$ (FIG. 2, bottom). Kleisins also recruit the 


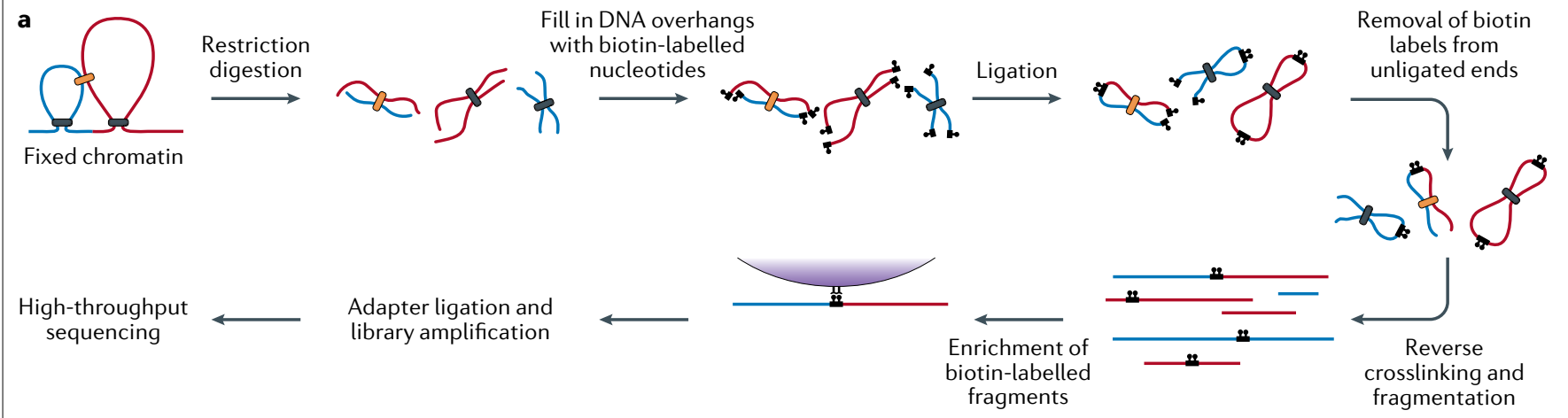

b
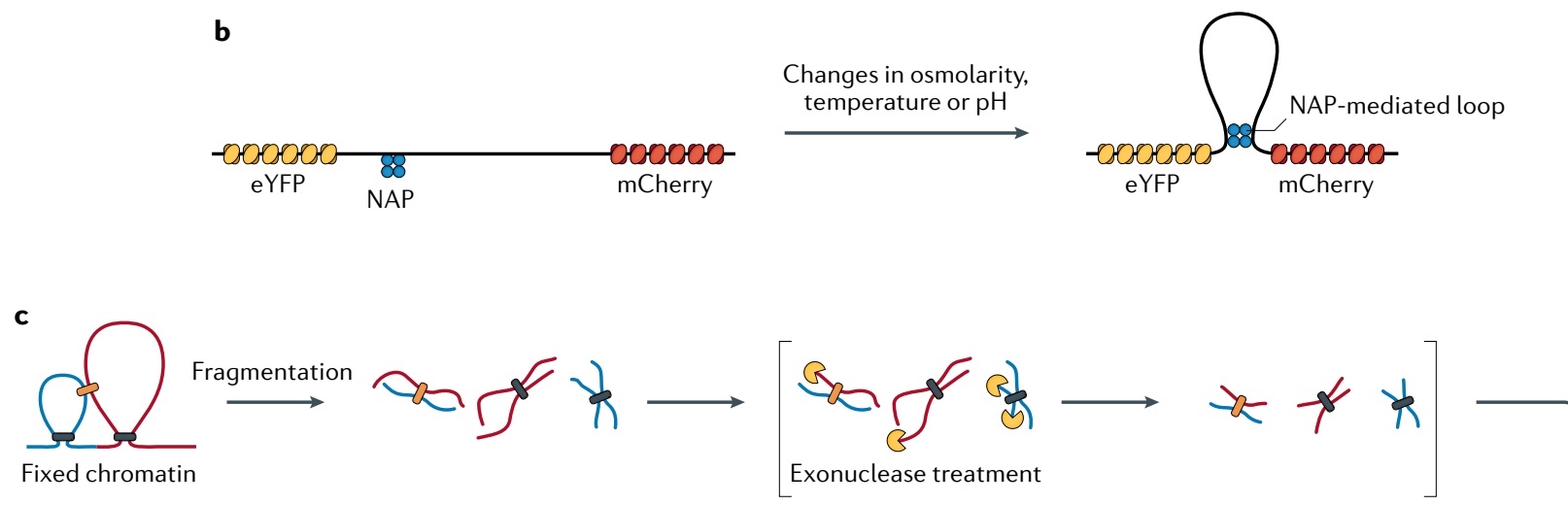

High-throughput

Adapter ligation and

library amplification
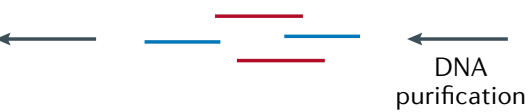

Immunoprecipitation

sequencing

\section{$\mathrm{Hi}-\mathrm{C}$}

$\mathrm{Hi}-\mathrm{C}$ is a chromosome conformation capture (3C)-based method used to study the three-dimensional organization of the chromosome ${ }^{176-178}$. It is a high-throughput technique that determines the probability of interaction between pairs of genomic loci in an unbiased manner at resolutions of up to $1 \mathrm{~kb}\left(\mathrm{REFS}^{90,177}\right)$. Hi-C allows the study of chromosome organization in situ in the nucleus or nucleoid, and changes therein in response to environmental stimuli, and - by alignment with genome-wide proteinoccupancy profiles such as chromatin immunoprecipitation (ChIP) data sets - identification of proteins involved in chromosome structuring $38,44,80,81,85,89-91,177$. Hi-C is generally an ensemble technique providing an averaged chromosome interaction profile. Single-cell $\mathrm{Hi}-\mathrm{C}$ is gaining momentum in the field of chromosome biology, yet has still to be applied to bacterial organisms ${ }^{179-181}$.

The technique involves treating cells in culture with formaldehyde to chemically crosslink all DNA-protein and protein-protein interactions within the cell, hence fixing the structure of the chromosome (see the figure, part a). Of the remaining steps outlined in the figure part a, the key principle is that loci that are close to each other in three-dimensional space are ligated into individual DNA-ligation products regardless of their position along the primary genome sequence. Ligation products are read out using high-throughput sequencing to identify interacting pairs of genomic loci en masse. The sequencing data are represented as a heatmap of genome-wide interaction probabilities.

\section{Fluorescent repressor operator system}

The fluorescent repressor operator system (FROS) is a microscopybased technique used to determine the position and track the dynamics of specific loci in living cells. Loci of interest are marked with an array of 'operator' sequences that can be recognized and bound by ectopically expressed 'repressor' proteins that are translationally fused to fluorescent protein labels (see the figure, part b). Different loci can be tagged and independently tracked in live cells to determine the spatial position and inter-relationship of positions, establishing changes in structure in response to environmental stimuli. Changes in chromosome structure are evident as changes in the distance between pairs of loci. FROS is a single-cell technique that reveals nonaveraged chromosome dynamics. Despite limitations in its throughput, it is more powerful in establishing direct structure-function relations than $\mathrm{Hi}-\mathrm{C}$.

\section{Chromatin immunoprecipitation}

ChIP uses antibodies that target a DNA-binding protein of interest to isolate the factor and the chromosomal regions associated with it following enzymatic, chemical or physical genome fragmentation. Often combined with high-throughput sequencing (ChIP-seq), the technique measures genome-wide patterns of DNA binding at single-base-pair resolution.

In this method, the chromosome of cells in culture is fixed with formaldehyde to crosslink all protein-protein and protein-DNA interactions (see the figure, part c). The chromosome is fragmented and antibodies are used to immunoprecipitate any DNA segments that are bound by the protein of interest. In ChIP-seq, the immunoprecipitated library is then purified and sequenced to determine the genome-wide DNA-binding profile of the protein of interest.

eYFP, enhanced yellow fluorescent protein; NAP, nucleoid-associated protein. 
a

SMC complexes load at ParB-bound parS b

$\rightarrow \quad \begin{gathered}\text { Bidirectional loop } \\ \text { extrusion }\end{gathered} \quad \rightarrow$

c

SMC complexes migrate towards Ter d

Oppositely orientated HEG slows loop extrusion
One-ring, twoDNA model

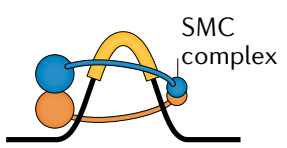

Handcuff model

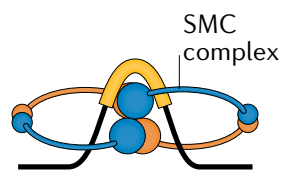

ParB-bound
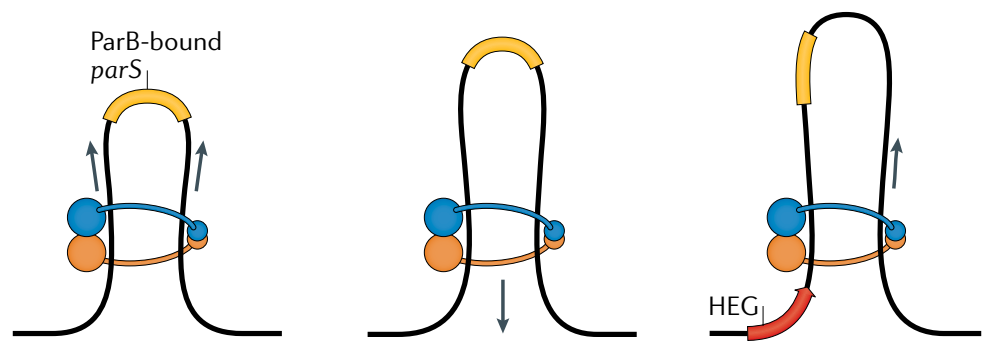

ParB-bound
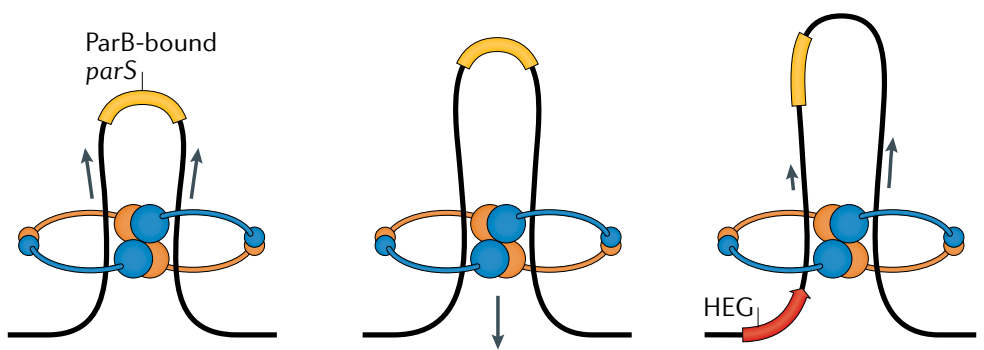

Fig. 2 I SMC proteins function as loop-extruding factors. a | The structural maintenance of chromosomes (SMC) complex is loaded onto the DNA at ParB-bound parS sites in bacteria ${ }^{37-39} \cdot \mathbf{b}$ | The SMC complex then bidirectionally 'pulls' the DNA through its ring to extrude a growing, unknotted loop of DNA ${ }^{40,42,43}$. The loop may be formed by an SMC complex entrapping two DNA strands within a single ring (one-ring, two-DNA model (top)) or by a dimer of two SMC rings that each trap one DNA segment (handcuff model (bottom)). c | Loop extrusion allows bacterial SMC complexes to progressively move from the ParB-bound parS sites that are positioned close to the origin of replication towards the terminus (Ter) region ${ }^{38,44}$. $\mathbf{d}$ | The progression of SMC along the DNA is slowed by convergent transcription ${ }^{38,44}$ and may be slowed by up to $80 \%$ on encountering an oppositely oriented highly expressed gene (HEG) ${ }^{44}$.

Nucleoid-associated proteins

(NAPs). A broad term to describe any proteins implicated in organizing bacterial chromosomes. Here we consider structural maintenance of chromosomes (SMC) proteins as NAPs due to their association with the nucleoid and their role in shaping nucleoid structure. SMC proteins - discovered later than other NAPs and initially studied primarily in the context of chromosome segregation - have historically (and in our view unjustly) not been classified as NAPs.

Nucleoprotein

A generic term, applicable to prokaryotes and eukaryotes, to describe DNA in complex with bound proteins. kite and hawk proteins. Bacterial and archaeal SMCkleisin complexes, and the eukaryotic Smc5-Smc6 complex, recruit the kite proteins. The condensin and cohesin SMC complexes of eukaryotes recruit the hawk proteins $^{20,22}$.

In bacteria, three classes of SMC family proteins have been identified: SMC-ScpAB in B. subtilis and Caulobacter crescentus, SMC-like MukBEF in E. coli and other gammaproteobacteria and deltaproteobacteria, and the MukBEF-like MksBEF, which has been detected in a wider range of bacterial species. These SMC family proteins are involved in segregation of newly replicated sister chromosomes ${ }^{27-36}$. SMC-kleisin complexes are loaded onto the chromosome at the centromere-like parS sequences, positioned close to the origin of replication, by the parS-binding protein, $\operatorname{ParB}^{37-39}$. Loading factors for the SMC-like proteins MukBEF and MksBEF are currently unknown. Once associated with DNA, SMC complexes generate and maintain DNA loops, and are mechanistically characterized as loop-extruding factors $^{40,41}$ (FIC. 2). By contrast, there is currently no evidence to suggest that SMC-like MukBEF and MksBEF play the same role. First proposed in $2001\left(\right.$ REF $\left.^{42}\right)$, and formalized theoretically in $2012\left(\mathrm{REF}^{43}\right)$, loop extrusion involves the clamping of the protein complex around contiguous DNA sequences $^{42}$ (FIG. 2a). The factor then 'pulls' the DNA through the clamp to produce a growing, unknotted loop of $\mathrm{DNA}^{40}$ (FIG. 2b). SMC proteins bidirectionally extrude DNA and progressively move along the chromosome ${ }^{43}$ towards the terminus (FIG. 2b,c). The SMC extrusion complex may consist of a pair of DNA molecules pulled through one SMC ring, referred to as the 'one-ring, two-DNA model' 25 (FIG. 2, top), or one DNA molecule pulled through each ring of a 'handcuffed' pair $^{26}$ (FIG. 2, bottom). The rate of loop extrusion by SMC is affected by transcription. Oppositely oriented highly expressed genes (HEGs) attenuate the progression of $\mathrm{SMC}^{38,44}$ (FIG. 2d). For instance, in B. subtilis, SMC progression can be slowed by more than $80 \%$ due to an oppositely oriented $\mathrm{HEG}^{44}$. As with other DNA-looping proteins, SMC proteins have been suggested to function by static loop formation - stably anchoring a pair of DNA loci to form a loop ${ }^{45}$. However, as supporting evidence that SMC proteins largely function by active loop extrusion in bacteria, chromosome arms progressively align from the origin to the terminus on replenishment of the SMC loading factor ParB in B. subtilis ${ }^{44}$, but they aberrantly align upon repositioning parS sites in C. crescentus $^{38}$.

DNA bending by IHF, HU and Fis. Whereas H-NS and SMC proteins manage DNA loops ${ }^{46}$, other NAPs primarily bend the DNA. For example, the factor for inversion stimulation (Fis) binds DNA as a dimer (FIG. 1d) by virtue of a helix-turn-helix motif ${ }^{47}$. Fis recognizes a 15-bp degenerate DNA palindrome characterized by a $\mathrm{G}$ at position 1 
and a Cat position 15 (5'-GNNVRWWWWWYVNNC-3'). Target recognition is driven by the shape of the minor groove resulting from the binding site sequence rather than the sequence itself ${ }^{47}$. The degree of DNA bending induced by Fis binding can vary between $50^{\circ}$ and $90^{\circ}$ (FIG. 1d) depending on the flanking DNA sequence ${ }^{48}$. Fis is often found at points where DNA duplexes cross ${ }^{49,50}$. This may stabilize plectonemes in supercoiled DNA.

DNA bending by integration host factor (IHF) is more severe, generating $160^{\circ}$ bends ${ }^{51}$ (FIG. 1 e). IHF binds its consensus sequence ( $5^{\prime}$-WATCAANNNNTTR- $\left.3^{\prime}\right)^{52}$ as a heterodimer, composed of $\alpha$-subunits and $\beta$-subunits. The minor groove is contacted by a $\beta$-ribbon arm that protrudes from each subunit of the heterodimer (FIG. 1e). This interaction is favoured by A-tracts. The insertion of a proline residue at the tip of each $\beta$-arm into the DNA base stack induces a hairpin bend by kinking the DNA on either side of the hairpin ape ${ }^{51}$. High intracellular concentrations of IHF permit non-specific interactions with many non-specific DNA targets, probably in a manner similar to heat-stable protein from E. coli strain U93 (HU) (see below). The protein IHF has been identified only in Gram-negative bacteria.

HU shares $40 \%$ sequence identity with IHF subunits $^{53}$. Unlike IHF, HU is found widely distributed among bacteria $^{54}$. In $E$. coli, HU forms $\alpha$-subunit and $\beta$-subunit heterodimers. However, HU homodimers predominate across other bacteria, in which a single gene encoding $\mathrm{HU}$ is often present. HU has no sequence specificity, but its mode of target recognition is similar to that of IHF. DNA is bent to a lesser extent by HU than IHF and over a range of different angles - akin to a flexible hinge ${ }^{55,56}$. Binding of $\mathrm{HU}$ also occurs preferentially at naturally bent or distorted $\mathrm{DNA}^{57}$. The bends induced by each $\mathrm{HU} \beta$-arm force the DNA out of a single angular plane $e^{55,58}$. Consequently, sequential binding of $\mathrm{HU}$ dimers induces coiling of the DNA around the bound proteins to form filaments. This means that $\mathrm{HU}$ can restrain negative supercoils in DNA and, alongside topoisomerase I, introduce negative supercoils in circular DNA ${ }^{59-61}$. Generally, DNA is negatively supercoiled in bacteria to facilitate DNA transactions that require DNA melting ${ }^{62}$.

DNA loops in which the double-stranded DNA is wrapped around itself as a result of supercoiling.

Supercoiled

Pertains to supercoiling, which is underwinding or overwinding of the double helix that causes the double-stranded DNA to fold into higher-order

structures: plectonemes and toroids. To alter DNA

supercoiling levels, enzymatic breaking and rejoining of DNA strands is required.

\section{Topoisomerase}

An enzyme that alters DNA supercoiling by breaking and rejoining DNA strands.

Mechanistically, topoisomerases are distinguished by whether they break and rejoin either a single strand (type I) or both strands (type II). as hilA, with minimal disruption of the H-NS binding profile at the operon $^{69}$.

Comparison with eukaryotic and archaeal DNA-folding proteins. The nomenclature for NAPs (for instance, 'histone-like nucleoid structuring protein' for H-NS) can imply a relationship to eukaryotic histones. However, there are few similarities at the protein level. Most notably, H-NS is histone-like in only one regard: it is an abundant DNA-binding protein. Even so, there are many examples of eukaryotic DNA-binding proteins that utilize arginine hooks to bind AT-rich $\mathrm{DNA}^{70}$. These can have global DNA-folding properties. For example, the metazoan special AT-rich sequence-binding protein 1 (SATB1) has genome-wide roles in DNA folding and, like H-NS, might link higher-order nucleoprotein structures and gene regulation ${ }^{71-73}$. HU, like histones, is able to induce DNA supercoiling ${ }^{59-61}$. Indeed, the ability of HU to wrap DNA in filaments hints that the protein has the capacity to form structures similar to hypernucleosomes in archaea ${ }^{74,75}$. However, although the proteinDNA co-crystal structures are comparable for archaeal histones and HU, results from solution studies do not support this model ${ }^{56}$. Structurally and functionally, SMC complexes in bacteria and eukaryotes have similar functions in managing DNA loops (see later).

Analogously to eukaryotic histones, bacterial NAPs also undergo post-translational modifications. To date, 29 post-translational modifications have been identified for E. coli $\mathrm{H}$-NS that may fine-tune its properties. Acetylation neutralizes charges of Lys83, Lys87, Lys96, Lys120 and Lys121, which are known to facilitate DNA binding. Succinylation of Lys96, Lys 120 and Lys 121 may also interfere with DNA binding due to steric hindrance $^{76-79}$. Some HU proteins have terminal extensions enriched in lysine, proline or alanine repeats, reminiscent of the (S/T)PKK motifs found in eukaryotic histone $\mathrm{H} 1$ that are subject to post-translational modification ${ }^{54}$.

\section{Local patterns of DNA folding}

Chromosome interaction domains. At a scale of tens to hundreds of kilobases, the bacterial chromosome (FIG. 3Aa) is partitioned into chromosome interaction domains (CIDs) ${ }^{80,81}$ (FIG. 3Ab), which are analogous to the topologically associating domains (TADs) in eukaryotes $^{82-84}$ (compare FICS $3 \mathrm{Ab}$ and $3 \mathrm{Bb}$ ). CIDs and TADs exhibit a high degree of self-interaction and are insulated from flanking regions.

$\mathrm{Hi}-\mathrm{C}$ in C. crescentus indicates that the chromosome is organized into 23 CIDs during exponential growth in rich medium and 29 CIDs in starvation conditions, with the length of these domains ranging between 30 and $420 \mathrm{~kb}\left(\mathrm{REFS}^{80,85}\right)$. The boundaries between CIDs correspond to positions of HEGs that are more than $2 \mathrm{~kb}$ in length ${ }^{80,85}$ (FIG. 3Ab). In C. crescentus, these include, for instance, genes within the ATP synthase and $\mathrm{NADH}$-quinone oxidoreductase gene clusters during exponential growth in rich medium, and starvation-induced genes such as CCNA_03169 (which encodes an Lrp-like asnC family transcription regulator) and CCNA_03327 (which encodes a histidine kinase involved in signal transduction) during periods 
Aa

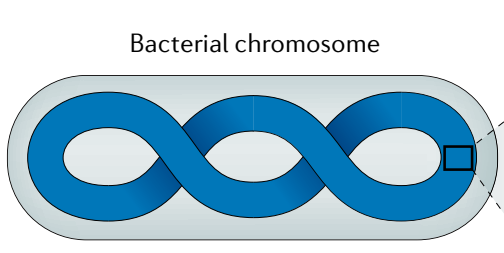

Ab

Chromosome interaction domains
Ac

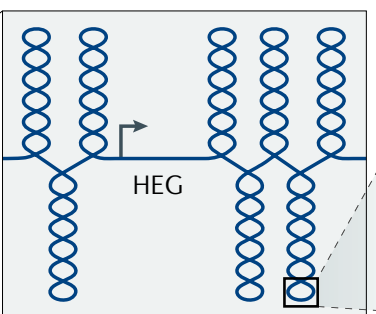

Operon-level loops

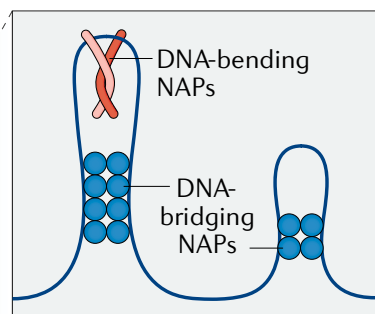

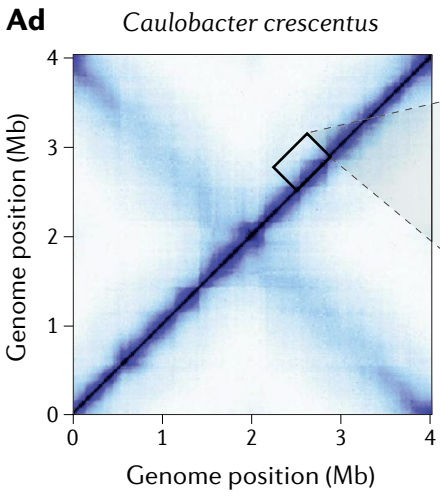

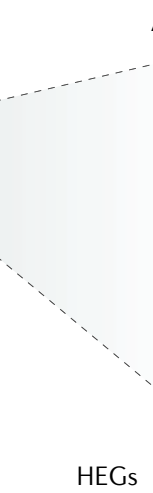

Ae

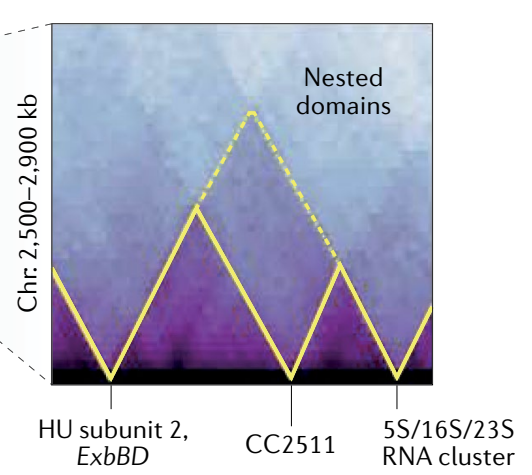

Ba

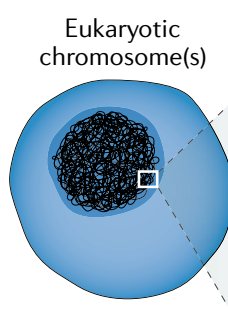

Bb

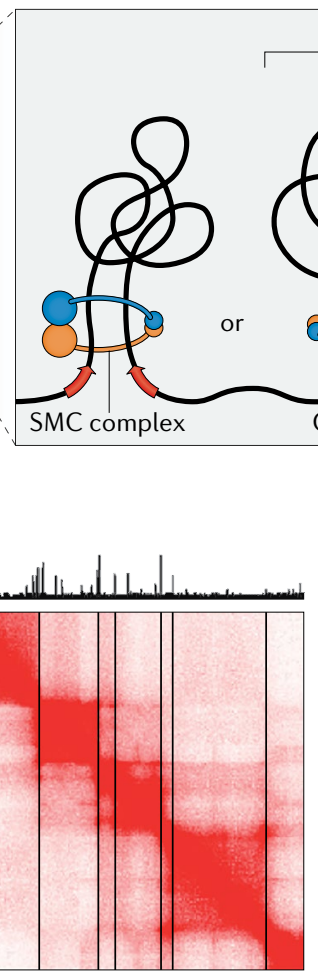

Bd

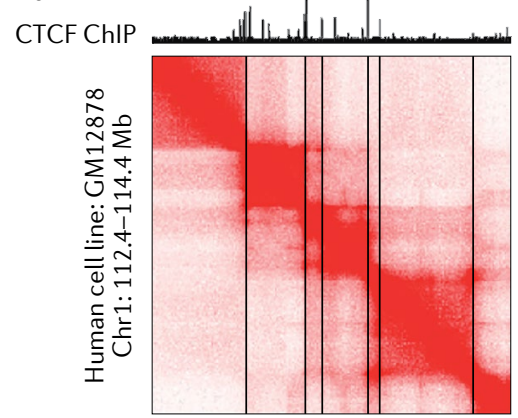

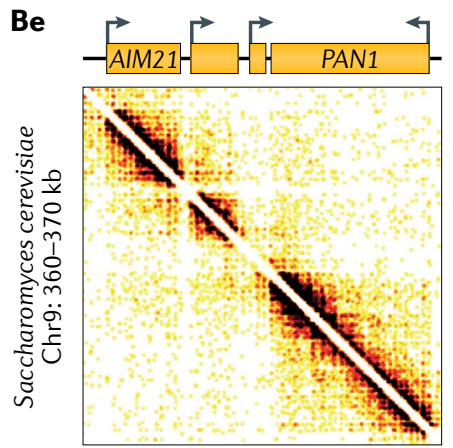

the genes in the cluster exhibit a high transcription rate. During starvation, in accordance with the decreased rate of gene expression within the cluster, the sharpness of the boundary diminishes ${ }^{80}$. Recombination-based experiments indicate that while HEGs generally form 
4 Fig. 3 | Chromosomes are hierarchically organized in bacteria and eukaryotes. A | Bacterial chromosome organization. At a global scale, the bacterial chromosome is spirally folded to fit within the bacterial cell (part Aa). Regions of the chromosome sequentially close to each other interact in three-dimensional space as evidenced by the presence of a primary diagonal of high interaction frequency in a $\mathrm{Hi}-\mathrm{C}$ contact map (part Ad). Except for Escherichia coli, all reported bacterial chromosome interaction profiles also show a secondary diagonal of low interaction frequency that lies perpendicular to the primary diagonal (part Ad). This feature indicates interaction between the chromosome arms that run alongside each other in the spirally organized chromosome (interarm interaction) ${ }^{80,81,86,101}$. At the scale of tens to hundreds of kilobases, the chromosome is subdivided into chromosome interaction domains (CIDs) (part Ab) ${ }^{80,81}$ CIDs exhibit self-interaction and are insulated from flanking chromatin. These structures are observed as squares along the primary diagonal of a $\mathrm{Hi}-\mathrm{C}$ map (part Ad) or as triangles when observing one half of the symmetric Hi-C map (part Ae). Bacterial CIDs are nested $^{80,81,86}$ : larger domains (broken yellow line) are organized into smaller subdomains (solid yellow line) (part Ae $)^{80}$. The boundaries between the domains are typically formed by highly expressed genes (HEGs) more than $2 \mathrm{~kb}$ in length that physically separate the flanking chromatin (part $\mathbf{A b})^{80,85}$. The smallest structural unit of organization of the bacterial chromosome may correspond to loops formed at the level of individual operons by nucleoid-associated proteins (NAPs) (part Ac). B | Eukaryotic chromosome organization. The eukaryotic chromosome, localized inside the nucleus (part $\mathbf{B a}$ ), is organized into topologically associating domains (TADs) (part $\mathbf{B b}$ ), which are analogous to the bacterial CIDs. TADs are formed by loop extrusion ${ }^{43,88,89}$. Eukaryotic structural maintenance of chromosomes (SMC) proteins load onto the chromosome and extrude DNA loops (FIG. 2) until the complexes collide with inward-facing CCCTC-binding factor (CTCF)-binding sites (part $\mathbf{B b})^{43,88,89}$. TAD boundaries — identified as the region between two squares along the diagonal of a $\mathrm{Hi}-\mathrm{C}$ matrix - occur at genomic regions enriched in CTCF (part Bd) ${ }^{90-92}$. Eukaryotic TADs are nested, with the smallest sub-TAD interaction domains typically comprising up to five genes (parts Bc,Be) ${ }^{96}$. ChIP, chromatin immunoprecipitation; $\mathrm{HU}$, heat-stable protein from $\mathrm{E}$. coli strain U93. Hi-C contact maps in parts Ad and $\mathbf{A e}$ are adapted with permission from REF. ${ }^{80}$, AAAS. GM12878 Hi-C map in part Bd is adapted with permission from REF. ${ }^{91}$, Elsevier. Saccharomyces cerevisiae Micro-C map in part $\mathrm{Be}$ is adapted with permission from REF. ${ }^{96}$, Elsevier.

plectoneme-free regions that act as supercoil diffusion barriers, only long HEGs can generate extended supercoil diffusion barriers that insulate CIDs by physically separating flanking chromatin ${ }^{85}$. Indeed, the ectopic insertion of a long HEG is sufficient to establish a CID boundary in the chromosome ${ }^{80}$.

The B. subtilis chromosome is organized into 20 CIDs $50-300 \mathrm{~kb}$ in length. While $60 \%$ of the CID boundaries coincide with HEGs, $30 \%$ overlap with sections of the genome bound by the protein $\operatorname{Rok}^{81}$. This observation implies that Rok (and, by extrapolation, other bacterial NAPs) could function as domain barriers. The E. coli chromosome appears to be organized into $31 \mathrm{CIDs}$ between $40 \mathrm{~kb}$ and $\sim 300 \mathrm{~kb}$ in size. Twenty-two of the CID boundaries correspond to the positions of HEGs, and nine boundaries coincide with positions of genes that code for proteins with an export signal sequence ${ }^{86}$. This may be relevant in light of the hypothesis that chromosomes are membrane appended by coupled transcription-translation-translocation ${ }^{87}$. While multiple systems contribute to the formation of CID boundaries in bacterial chromosomes, the hierarchical structural organization that they contribute to is conserved. Bacterial CIDs exhibit a nested domain organization with each domain composed of smaller subdomains ${ }^{80,81,86}$ (FIG. 3Ad,Ae). The smallest

Replichores

The sections of a chromosome between the origin and the

terminus of replication. Circular chromosomes are usually divided into a left replichore and a right replichore. condensin and Smc5/6 function as loop-extruding factors. Unlike their bacterial counterparts, eukaryotic SMC complexes do not appear to be loaded onto the chromosome/chromatin at a specific DNA sequence. On clamping around the DNA, eukaryotic SMC complexes continue to extrude loops until the complexes spontaneously dissociate from the DNA, collide with another factor or encounter an appropriately oriented TAD boundary element ${ }^{43,88,89}$. TAD boundaries are encoded in the genome as CCCTC-binding factor (CTCF)binding sites. These sites exhibit a directionality, such that a TAD forms only between a pair of inward-facing CTCF sites ${ }^{90-92}$ (FIG. 3Bb). Indeed, deletion or inversion of CTCF-binding sites disrupts TAD boundaries in vivo ${ }^{89}$. Curiously, in Drosophila melanogaster, SMC complexes and CTCF are not markedly enriched at TAD boundaries. In flies, this role is played by the insulator complexes BEAF32-CP190 and BEAF32-Chromator ${ }^{93-95}$.

As in bacteria, the TADs of eukaryotic genomes are nested domains ${ }^{90,94,96}$. The smallest organizational units of TADs correspond to individual genes in Saccharomyces cerevisiae ${ }^{96}$ (FIG. 3Be). The functional relevance of this organization and the molecular determinants of the boundaries are still unclear.

The impact of DNA supercoiling. Local patterns of DNA supercoiling influence DNA folding within topologically isolated regions of the E. coli chromosome (FIG. 3Ab). However, tools to measure chromosome-wide patterns of DNA folding have become accessible only in recent years $^{97,98}$. As noted already, DNA is in an average state of negative supercoiling. However, supercoiling density is unevenly distributed and varies across phases of growth. In particular, a gradient of increased negative supercoiling runs from the origin of replication to the terminus, along each arm of the chromosome, only in starved cells $^{97}$. This gradient requires the protein HU. The wrapping of DNA around HU and the change in twist of the double helix mediated by the protein are consistent with effects of HU on global DNA supercoiling ${ }^{97}$. The expression levels of HU also vary greatly across different phases of growth ${ }^{99}$, potentially explaining effects on DNA topology. Collectively, this may also explain why loss of HU has different effects on intrachromosome interactions in different bacteria ${ }^{80,86}$ with different levels of DNA supercoiling ${ }^{100}$.

Interactions between chromosome arms. In bacteria, progression of SMC from the origin to the terminus mediates contacts between the right and left replichores, resulting in their parallel alignment. This manifests itself as a characteristic 'secondary diagonal', perpendicular to the main diagonal in $\mathrm{Hi}-\mathrm{C}$ matrices of bacterial chromosomes $^{80,81,101}$ (FIG. 3Ad). Curiously, this secondary diagonal is absent in the contact maps of the E. coli chromosome ${ }^{86}$ despite the presence of the SMC-like MukBEF system. The MukBEF complex, in the absence of ATP, consists of a V-shaped MukB dimer, the MukF kleisin, which extends between the pair of MukB head domains, and four MukE kite proteins. Unlike other characterized kleisins, MukF is not monomeric ${ }^{19,20,102}$. Instead, MukF forms a dimer via its $\mathrm{N}$-terminal winged-helix domain 
and binds MukB via its C-terminal domain ${ }^{103,104}$. On binding ATP, the MukB ATPase heads dimerize and sterically displace one of the two MukF monomers, rendering the $\mathrm{N}$ terminus of the MukB-bound MukF available for dimerization with another ATP-bound MukBEF complex ${ }^{104}$. The subsequently formed dimer is the minimal functional unit of MukBEF ${ }^{105}$. ATP-bound MukBEF stably associates with the chromosome and is involved in its condensation, while ATP hydrolysis results in MukBEF dissociation ${ }^{105,106}$. The hydrolysis of both ATP molecules contained within the dimerized head domains is required for a single MukBEF unit to release the DNA ${ }^{104-106}$. The MukBEF complex has been proposed to move along the chromosome as a 'rock climber'. In this model, ATP hydrolysis in a single unit of a MukBEF dimer releases it from the chromosome, while the other unit remains bound. ATP binding then allows the released MukBEF unit to capture a different segment of the DNA and hence move along the chromosome $^{105}$ (FIG. 4A). The release-capture cycles of this model imply that the minimal functional unit of MukBEF in E. coli cannot promote and maintain interarm interactions as observed for loop-extruding SMCs such as SMC-ScpAB in B. subtilis. Other models that consider that a MukBEF dimer is not completely released from DNA on ATP hydrolysis speculate that MukBEF may still carry a loop extrusion functionality ${ }^{107}$ (FIG. 4B).

\section{Global chromosome organization}

Over the past 10 years, advances in genome-scale approaches have improved our understanding of bacterial DNA folding at the micrometre scale (BOX 1). Most notably, chromosomal patterns of NAP binding and physical interaction frequencies have revealed independently organized macrodomains with distinct properties ${ }^{4}$. Such structures are best defined for $E$. coli, where the chromosome is divided into four macrodomains and two non-structured regions. All macrodomains exhibit reduced intracellular mobility compared with the nonstructured chromosomal regions. Thus, macrodomains tend to interact with the non-structured regions but not with other macrodomains ${ }^{108}$.

Constraint of Ori macrodomain mobility by MaoP. The Ori macrodomain contains the origin of chromosome replication oriC ${ }^{109,110}$. The constrained mobility of Ori requires the yifE gene product, MaoP (macrodomain Ori protein), and a 17-bp motif in the upstream intergenic region (5'-CTAATACTCCGCGCCAT-3') named maos (macrodomain Ori sequence) ${ }^{109}$. In otherwise wild-type cells, inactivation of maoS/MaoP specifically increased the mobility of $\mathrm{Ori}^{109}$. It is not known how MaoP acts over long distances to constrain DNA mobility.

Condensation of Ter macrodomain structure by MatP. The terminus (Ter) macrodomain is diametrically opposed to Ori (FIG. 5Aa) and encompasses the replication terminus. A major breakthrough for understanding Ter was the identification of a sequence repeated 23 times in Ter but not elsewhere in the E. coli chromosome $\left(5^{\prime} \text {-GTGACRNYGTCAC- } 3^{\prime}\right)^{111}$. The same sequence uniquely occurs in equivalent parts of many bacterial chromosomes ${ }^{111}$. This DNA site, named 'macrodomain Ter sequence' (matS) is the target of the macrodomain Ter protein (MatP). This interaction is highly specific, as shown by MatP exclusively binding these DNA targets in chromatin immunoprecipitation experiments ${ }^{111}$. Loss of MatP activity leads to decondensation of the Ter macrodomain ${ }^{111}$. MatP consists of three domains: an $\mathrm{N}$-terminal four-helix bundle, a central $\beta$-strandhelix-helix and a C-terminal coiled coil ${ }^{112}$. Interaction of MatP with DNA is mediated by the $\beta$-strand-helixhelix, which resembles ribbon-helix-helix structures found in other DNA-binding proteins ${ }^{112}$. MatP binds DNA as a dimer mediated by interactions involving both the N-terminal domain and the central domain. The C-terminal coiled coil is required for tetramerization of MatP. Such tetramers can generate bridges between distal matS sites on the chromosome, effectively condensing the Ter macrodomain ${ }^{112,113}$. This is evident in Hi-C experiments: deletion of matP specifically restructures Ter, with reduced intradomain interactions being observed ${ }^{86}$. Loss of MatP also prevents correct positioning of the DNA replication at midcell, and this depends on an interaction between MatP and division apparatus-associated protein $\mathrm{ZapB}^{114}$.

Other proteins with macrodomain-specific DNA-binding properties. In E. coli at least two additional proteins, SeqA and SImA, have macrodomain-specific DNAbinding properties ${ }^{115,116}$. However, unlike MaoP and MatP, there is no evidence that SeqA and SImA contribute to the overall folding of these domains. Briefly, SeqA is involved in sequestration of the DNA replication origin after a new round of DNA replication has been initiated ${ }^{117}$. This is permitted because newly replicated DNA is hemimethylated at $5^{\prime}$-GATC-3' motifs targeted by DNA adenine methylase (Dam) and SeqA ${ }^{118}$. These 5'-GATC-3' motifs are under-represented in the Ter macrodomain and over-represented elsewhere, particularly near the origin of replication ${ }^{116}$. Similarly, SlmA binds throughout the E. coli chromosome, except in the Ter macrodomain ${ }^{115}$. SlmA recognizes the sequence $5^{\prime}$-GTGAGTACTCAC- $3^{\prime}$ and is required for correct cell division $^{115}$. SlmA, SeqA, MatP and MaoP are co-conserved in bacteria encoding Dam methylase, suggesting that these bacteria use similar strategies to organize their chromosomes. Indeed, even in bacteria lacking Dam, proteins with similar patterns of chromosomewide DNA binding have been identified. For instance, in B. subtilis the nucleoid occlusion (Noc) protein appears to be the functional equivalent of $\operatorname{Sim} A^{119}$. Similarly, in C. crescentus, GapR targets a large region surrounding the origin of replication ${ }^{120,121}$.

\section{Environmental regulation}

The structure of the bacterial chromosome changes in response to the environment (FIG. $5 \mathrm{Ab}$ ). In part, this is because a small number of NAPs (most notably H-NS and MvaT) can undergo conformational changes in response to specific ligands ${ }^{63,122}$. More commonly, the intracellular concentration of NAPs alters in response to environmental triggers ${ }^{99}$. These two scenarios are discussed in more detail below. 
Aa

ATP-bound MukEF

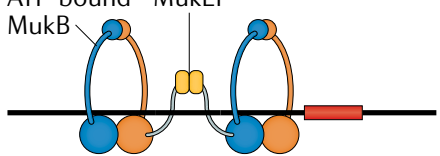

An ATP-bound dimer of dimeric MukBEF stably associates with the chromosome

Ad

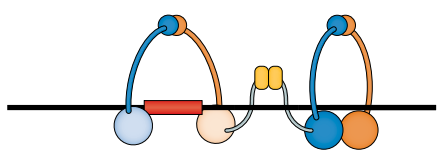

ATP hydrolysis in MukB heads of the second dimer releases it from the chromosome

Ba

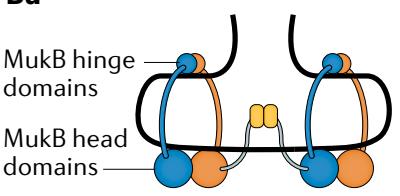

ATP-bound MukBEF binds DNA at MukB head and hinge domains
Ab

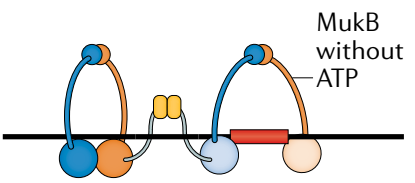

ATP hydrolysis in both MukB heads of a dimer releases it from the chromosome

Ae

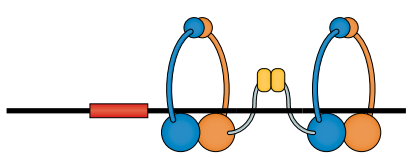

ATP binding re-establishes chromosome contacts

Bb

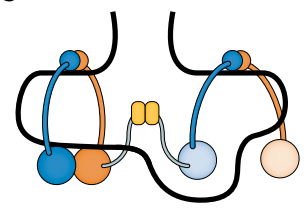

ATP hydrolysis in a MukB dimer releases the DNA from the head region, while it remains associated at the hinge
Ac

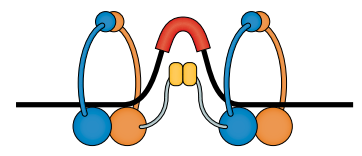

ATP binding re-establishes chromosome contacts

Af

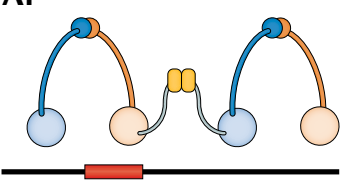

Simultaneous hydrolysis of all ATP molecules bound to MukBEF releases the complex

Bc

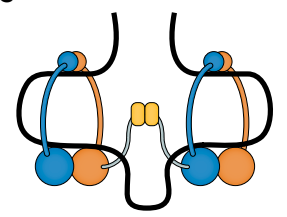

ATP binding re-establishes DNA contacts at a new DNA segment, extruding a DNA loop
Bd

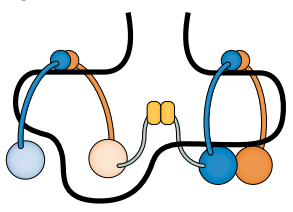

ATP hydrolysis in MukB heads of the second dimer releases the DNA from the head domain
Be

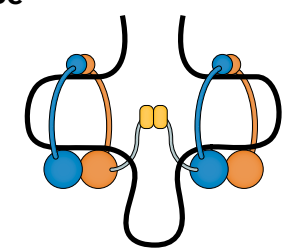

ATP binding re-establishes DNA contacts and enlarges the loop

Fig. 4 | MukBEF moves along the chromosome as a 'rock climber'. A | MukBEF movement along a single DNA molecule (based on the rock climber model proposed in $\mathrm{REF}^{105}$ ). Aa $\mid$ The minimal functional unit of the MukBEF complex corresponds to a dimer of dimers - $\mathrm{MukB}_{4} \mathrm{E}_{4} \mathrm{~F}_{2}$. When each of the MukB heads is bound to ATP, the MukBEF complex remains stably associated with the chromosome. Ab| Hydrolysis of ATP in both MukB heads of the same dimer disengages the MukB heads, and releases the dimer from the chromosome. The MukBEF complex remains bound to the DNA via the ATP-bound dimer. Ac | ATP binding to MukB of the released dimer re-establishes chromosome contacts with a different chromosomal locus. Ad,Ae |A DNA segment release and capture cycle in the second MukBEF dimer allows the complex to move along the chromosome as a 'rock climber'. Af | Simultaneous hydrolysis of all four ATP molecules bound to a MukBEF complex releases it from the chromosome. This step may involve a MukBEF 'unloading' factor. B $\mid$ MukBEF as a loopextruding factor ${ }^{107}$. Ba | ATP-bound MukBEF binds chromosomal DNA at the MukB head and hinge domains. Bb $\mid$ ATP hydrolysis in a MukBEF dimer releases the chromosomal DNA segment bound at the MukB head domains. Bc|ATP binding re-establishes MukB head-DNA contacts at a new DNA segment, thus generating a DNA loop. Bd,Be $\mid$ A release-capture cycle in the second MukBEF dimer results in loop enlargement, and hence loop extrusion.

Regarding environmentally triggered conformational changes of NAPs, helix $\alpha 3$ of $\mathrm{H}-\mathrm{NS}$ is unstable and frequently buckles. This folds one of the DNAbinding domains of the H-NS dimer onto the body of the protein ${ }^{63}$. A similar conformation is also adopted by the H-NS family protein MvaT under low-osmolarity conditions; electrostatic interactions occur between a positively charged patch at the C-terminal DNAbinding domain and a negatively charged patch at the $\mathrm{N}$-terminal domain ${ }^{122}$. In the folded conformation, one of the two DNA-binding domains of the protein dimer is unavailable for DNA binding, thus favouring the formation of lateral filaments along DNA ${ }^{63,122}$ (FIG. $1 \mathrm{~b}$, top). Magnesium ions stabilize helix $\alpha 3$ in $\mathrm{H}-\mathrm{NS}$ to prevent 
Aa

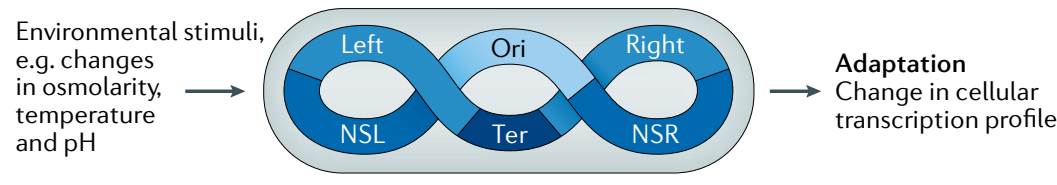

Ab Escherichia coli Exponential phase
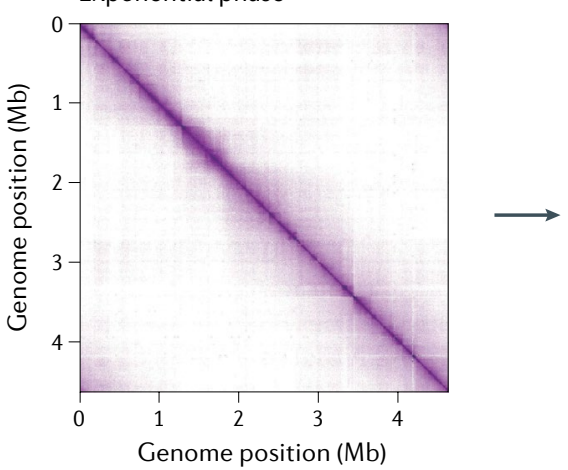

B

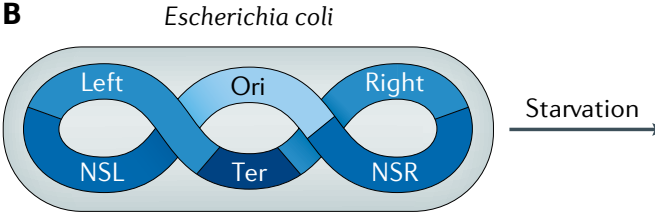

Compaction of the nucleoid decrease in cell volume
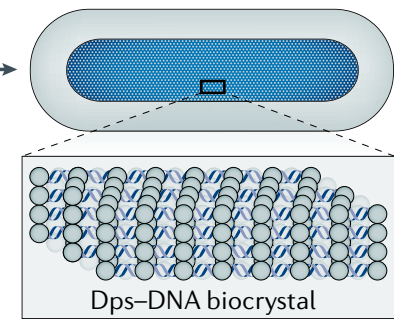

Fig. 5 | Environmental stimuli induce changes in chromosome organization. A|DNA reorganization in growing bacteria. Aa $\mid$ Reorganization of the bacterial nucleoid is induced in response to environmental stimuli such as changes in osmolarity, temperature and $\mathrm{pH}$. Consequently, activity of specific sets of genes required for environmental adaptation is altered. $\mathbf{A b} \mid$ The transition between the exponential and stationary phases of growth of Escherichia coli is associated with a reorganization of the bacterial chromosome. Specifically, the chromosome exhibits a weakening of compartmentalization into chromosome interaction domains. This is observed as 'blurring' of the squares along the main diagonal ${ }^{86}$. B $\mid$ Chromosome reorganization in starved bacteria. Reorganization of the bacterial nucleoid can be induced by starvation or stress. Such changes are apparent by light microscopy and indicate compaction of the nucleoid, often accompanied by a reduction in the overall cell volume ${ }^{127}$. The inset depicts the nucleoid structure in molecular detail as revealed by electron microscopy. Most notably, DNA-binding protein from starved cells (Dps; pale blue spheres) drives the formation of an ordered crystal lattice that incorporates DNA ${ }^{131}$. These structures are phase separated from other compartments of the cell and resistant to damage $\mathrm{e}^{50,133}$. NSL, left non-structured region; NSR, right nonstructured region; Ori, Ori macrodomain; Ter, Ter macrodomain. E. coli contact maps in part $\mathbf{A}$ are adapted with permission from REF. ${ }^{86}$, Elsevier.

buckling ${ }^{63}$. Correspondingly, high-osmolarity conditions destabilize the electrostatic interaction between the $\mathrm{N}$ - and C-terminal domains of $\mathrm{MvaT}^{122}$. Hence, both DNA-binding domains of the H-NS and MvaT dimers become available for DNA binding and bridged loops can form ${ }^{63,122}$ (FIG. 1 b, bottom). H-NS is also temperature sensitive. High temperatures reduce the cooperativity of $\mathrm{H}-\mathrm{NS}$ oligomerization and favour its dissociation from DNA $^{123-126}$.
A less subtle mechanism controlling chromosome dynamics is based on levels of NAP expression, which can change substantially. This is most notable during stress and starvation, when the nucleoid is reorganized into a condensed crystalline structure ${ }^{127}$ (FIG. 5B). Most NAPs are present at lower levels in starved cells, and Fis, which is among the most abundant DNA-binding proteins during periods of rapid cell division, is undetect$\mathrm{able}^{128}$. Conversely, while they are undetectable during rapid growth, DNA-binding protein from starved cells (Dps) and curved DNA-binding protein A (CbpA) accumulate to 175,000 and 14,000 copies per cell in stationary phase ${ }^{99,129}$. Both bind the DNA highly cooperatively, and interactions between DNA-bound protein molecules lead to DNA compaction ${ }^{50,130}$. Electron micrographs of Dps-DNA complexes reveal that they are organized in a crystalline lattice in vitro ${ }^{131}$ (FIG. 5B). A similar structure is observed in vivo when Dps is expressed in exponentially growing $\Delta$ fis strains ${ }^{131,132}$. Complexes of Dps or CbpA with DNA are resistant to damage induced by chemical and biological nucleases ${ }^{50,133}$. Hence, expression of these proteins is thought to protect the integrity of the genetic material in harmful environments. However, how these highly condensed protein-DNA structures coexist with other cellular processes has been a mystery. Recent work has shed light on the puzzle, revealing that supercondensed nucleoids of starved E. coli cells are phase-separated organelles ${ }^{133}$. Phase separation is an inherent physical property of macromolecules (such as proteins) to self-organize into condensates or 'droplets' in a crowded environment such as the cell interior. Binding of Dps to DNA in vitro blocks access to nucleases and hydroxyl radicals, but the DNA remains fully permissive to transcription ${ }^{129,133}$. This is because the physical properties of some molecules allows them to move between separated phases, while other molecules are trapped within a specific phase.

\section{Interplay with genome transactions}

As alluded to earlier, understanding how chromosome folding impacts other cellular processes has been a longstanding area of interest. Although Dps seems unable to impede transcription, the same is not true of other NAPs, which can have specific effects on gene expression. Furthermore, additional roles have been identified for NAPs in chromosome replication and segregation, as well as cell cycle progression. Here we describe the intricate interplay between NAPs, genome structure and diverse types of genome transaction.

Silencing of horizontally acquired genes by $H$-NS. H-NS targets DNA sequences that have a high AT content, often acquired by horizontal gene transfer. $\mathrm{H}-\mathrm{NS}$ binding at these loci represses transcription (known as xenogeneic silencing $)^{9}$. Remarkably, the majority of transcription suppressed by H-NS at such loci is spurious in nature ${ }^{134}$, arising from the high probability of sequences that fortuitously resemble promoter elements for RNA polymerase in high-AT-content DNA ${ }^{135,136}$. Left unchecked by H-NS, this transcription imposes a severe fitness defect due to titration of RNA polymerase and a global downshift in transcription of housekeeping genes ${ }^{134}$. 
Canonical gene regulation by $\mathrm{H}$-NS. Although most promoters repressed by H-NS have spurious output, $\mathrm{H}-\mathrm{NS}$ also plays a key role in regulating transcription of mRNAs. In these instances, the mechanisms by which $\mathrm{H}$-NS influences promoter activity appear diverse. A common mechanism of repression by $\mathrm{H}-\mathrm{NS}$ involves blocking the binding of RNA polymerase, or transcriptional activator proteins, completely ${ }^{137,138}$. Alternatively, at the $r r n B \mathrm{P} 1$ and $h d e A B$ promoters, $\mathrm{H}$-NS-induced DNA-looping traps RNA polymerase, interfering with promoter escape ${ }^{139,140}$. Similarly, but not involving loop formation, a direct contact between RNA polymerase and $\mathrm{H}-\mathrm{NS}$ can interfere with promoter clearance ${ }^{141}$. Because H-NS-controlled looping is mediated by environmental factors, many $\mathrm{H}$-NS-regulated genes are responsive to temperature and osmolarity. For instance, proVWX (the proU locus) is an H-NS-regulated osmosensitive operon. Its regulation requires two elements, the upstream regulatory element positioned upstream of the transcription start site and the downstream regulatory elements in the coding region that extends across the transcription start site $\mathrm{e}^{142-144}$. The two

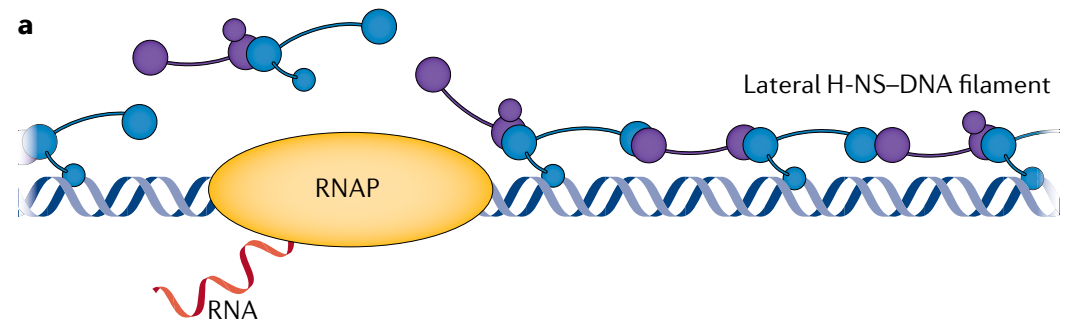

DNA-H-NS-DNA bridge
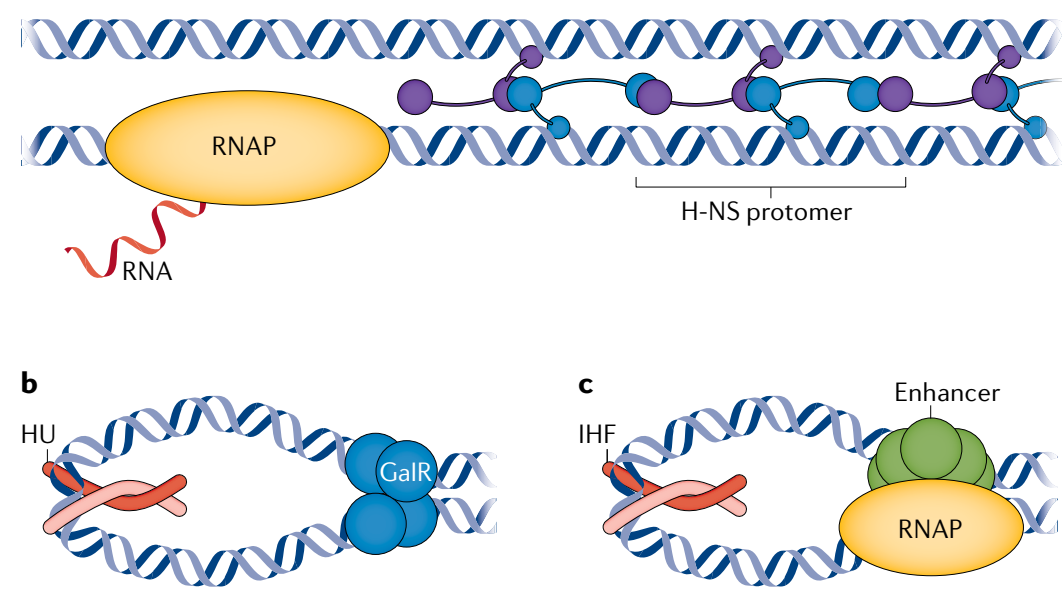

Fig. 6 | Modulation of transcription by nucleoid-associated proteins. a | Alternative types of histone-like nucleoid structuring protein (H-NS)-DNA complexes have different effects on transcription elongation. Top panel: lateral H-NS-DNA filaments can be invaded by RNA polymerase (RNAP). These H-NS-DNA complexes are unable to prevent transcription elongation and are either transiently displaced or remodelled as a result ${ }^{126,147}$. Bottom panel: bridged DNA-H-NS-DNA complexes are potent blocks to transcription and result in stalled elongation complexes ${ }^{126} \cdot \mathbf{b} \mid$ Stabilization of a DNA bend by HU facilitates repression. At the Escherichia coli gal operon the GalR repressor protein forms a repressosome that is stabilized by HU binding to the bent DNA ${ }^{154}$. c|Activation of transcription by DNA bending. Promoters that are dependent on enhancer-binding proteins for transcription require integration host factor (IHF) as a cofactor. The sharp DNA bend introduced by IHF brings the distally bound enhancerbinding protein into the proximity of RNAP so transcription can be activated ${ }^{157}$. $\mathrm{HU}$, heat-stable protein from $E$. coli strain U93. elements operate synergistically in H-NS-mediated osmoregulation $^{145}$. Such synergy could imply lateral or bridge-mediated interactions between the elements. Although direct evidence is lacking, in vitro experiments showing that only $\mathrm{H}$-NS-mediated bridging is sensitive to osmolarity lead us to hypothesize that $\mathrm{H}-\mathrm{NS}$ represses transcription of the proU operon by loop formation and that relief of repression involves local restructuring of the chromosome ${ }^{63,126}$. In pathogenic bacteria, H-NS can be utilized to control the expression of virulence factors during host colonization, with contributions from additional proteins that alter the ability of H-NS to multimerize and/or bind $\mathrm{DNA}^{146}$.

Regulation of transcription elongation by $H-N S$. As well as regulating the initiation of transcription, H-NS can control transcription elongation by impeding the progression of RNA polymerase. The ability of H-NS to hinder transcript extension depends on the type of H-NS-DNA complex. For example, RNA polymerase can transcribe genetic information through lateral H-NS-DNA filaments (FIG. 6a, top panel), whereas H-NS-DNA bridges efficiently block transcript extension and are likely to trap RNA polymerase in the loops formed ${ }^{126}$ (FIG. 6a, bottom panel). In both cases, it is not known if RNA polymerase advancement removes $\mathrm{H}-\mathrm{NS}$ from the DNA or if the nucleoprotein complex is transiently remodelled ${ }^{147}$.

Roles of bacterial H-NS in regulating transcription versus eukaryotic nucleosomes. In summary regarding the transcriptional roles of $\mathrm{H}-\mathrm{NS}$, loci bound by $\mathrm{H}-\mathrm{NS}$ are often not permissive to binding of RNA polymerase or regulatory proteins but can be remodelled for transcription to occur. By analogy, in eukaryotes, nucleosomes block transcription initiation, and so promoters are usually nucleosome-free. Histone modifications lead to remodelling of chromatin that impacts transcription ${ }^{148}$. For H-NS, transcription itself could lead to local remodelling of the nucleoprotein complex ${ }^{147}$. Furthermore, 'antisilencing' transcription factors can perturb repressive nucleoprotein filaments or interfere with their formation ${ }^{5}$.

Activation and repression of specific promoters by Fis. In general, Fis activates the expression of genes encoding products that are important for rapid cell division ${ }^{149}$. Conversely, Fis is often a repressor of genes that allow utilization of alternative carbon sources or terminal electron acceptors ${ }^{150,151}$. The DNA-folding activity of Fis appears to be important for counteracting the supercondensation of chromosomes mediated by $\mathrm{Dps}^{132}$. Taken together with the gene regulatory roles of Fis, this implies the protein is crucial to prepare cells for maximal rates of growth on exiting periods of starvation. This is consistent with observations that Fis is present at detectable levels only when cells are dividing rapidly ${ }^{128}$. The ability of Fis to activate or repress transcription is dependent on the position of binding and on interactions with other regulators at a given promoter. Hence, the mechanisms by which Fis activates 
and represses transcription are similar to those used by canonical transcription factors. For instance, to activate transcription of ribosomal RNA operons, Fis facilitates the recruitment of the transcriptional apparatus via a specific contact with the C-terminal domain of the RNA polymerase $\alpha$-subunit ${ }^{152}$. The same contact is made by many canonical transcriptional activators ${ }^{153}$. Similarly, the mechanisms of transcription repression by Fis are not unusual and involve occlusion of RNA polymerase or transcription factors ${ }^{150,151}$.

Stabilization of DNA repression loops by HU. Although it is unable to recognize specific DNA sequences, $\mathrm{HU}$ can regulate transcription from specific promoters. This results from the ability of HU to bind and stabilize certain deformations in DNA. This behaviour has been described for the E. coli galactose operon regulatory region. Two promoters at this locus are repressed by the activity of the repressor protein GalR. Maximal repression by GalR is mediated by interactions between GalR molecules bound at distal sites to create a repression loop. HU binds at the apex of the DNA loop and stabilizes the nucleoprotein complex, thus enhancing repression $^{154}$ (FIG. 6b). As HU affects global patterns of DNA supercoiling, genes responsive to DNA topology are part of the HU regulon ${ }^{155,156}$.

IHF can regulate transcription by bending the DNA. Like Fis, IHF is able to activate and repress transcription by binding to specific sites near promoters. This can be due to the ability of IHF to sharply bend DNA. For instance, IHF binds upstream of many E. coli promoters dependent on the alternative $\sigma$-factor, $\sigma^{54}$. $\sigma$-factors are general transcription factors (functionally similar to those found in eukaryotes) that are used by bacterial RNA polymerases to bind selectively to specific promoters. By bending the DNA, IHF facilitates interactions between RNA polymerase and enhancer proteins bound upstream. This stimulates promoter opening to activate transcription ${ }^{157}$ (FIG. 6c). The binding and bending of DNA by IHF can also repress transcription. In one example, at the $E$. coli $n r f$ promoter, IHF DNA binding alters interactions with a bound activator to hinder transcription activation ${ }^{158}$. The role of IHF as an activator or repressor depends on local nucleoprotein organization. Hence, there is no universal position upstream of a promoter from which IHF consistently exerts an activating versus repressive effect on transcription.

Interplay with replication and chromosome segregation. Chromosome architecture and NAPs also influence chromosome replication and segregation. The bacterial equivalent of the mitotic apparatus, the ParAB-parS partitioning system, and SMC proteins that are proposed to regulate origin firing in $B$. subtilis ${ }^{81}$ are evidently involved in the segregation of bacterial chromosomes into opposite cell halves concomitantly with replication $^{159-161}$ (FIG. 7A). In C. crescentus and B. subtilis, the ParB partitioning protein binds to the parS sequences present close to oriC to form a nucleoprotein complex on both sister chromosomes (FIG. 7A). The ParA ATPase is recruited to the complex and generates the free energy required for the resolution of the sister origins and their segregation $^{162}$. The loop-extruding complex SMC$\mathrm{ScpAB}$ is also recruited to the origin of replication by $\operatorname{ParB}^{33,37,39}$, from where it migrates along the chromosome to the terminus, extruding disentangled DNA of a single chromosome. This structurally separates sister chromosomes and favours their segregation ${ }^{38,44,81}$ (FIG. 7A).

The ParAB-parS partitioning system is absent in some species of gammaproteobacteria and deltaproteobacteria. In these organisms, the SMC-like MukBEF complex participates in chromosome segregation. In E. coli, MukBEF complexes containing an ATP-bound MukB are recruited to the origin ${ }^{34,163,164}$. MukB, in turn, recruits topoisomerase IV, a type II topoisomerase, which decatenates replicated sister origins ${ }^{165,166}$. Immediately after decatenation, the segregated origins, and the associated MukBEF clusters, move towards the quarter positions of the cell ${ }^{34,105}$ (FIG. 7B). Computational modelling suggests that segregation is driven by a selforganizing gradient of MukBEF and the origin of replication $^{167}$. MukBEF is also recruited to matS sites in the Ter macrodomain. At these sites, MatP and ATP hydrolysis by MukB release MukBEF complexes and associated topoisomerase IV enzymes. In $\triangle m a t P$ strains and strains with an ATPase-defective MukB, MukBEF accumulates in the Ter macrodomain, where it recruits topoisomerase IV and promotes early resolution of the chromosome terminus ${ }^{168}$.

Interplay with cell cycle progression. GapR, a conserved NAP of alphaproteobacteria, is a master regulator of cell cycle progression. Its binding sites overlap with loci bound by other regulators of cell cycle progression, including CtrA, MucR1, MucR2 and GcrA ${ }^{169}$. GapRdepleted and $\triangle g a p R$ strains of $C$. crescentus are temperature sensitive and exhibit cell division defects, forming filamentous, undivided cells or anucleate cells ${ }^{120,121,169}$. GapR binds to the origin of replication of the C. crescentus chromosome, where it is involved in the initiation of replication ${ }^{121}$. GapR also binds DNA ahead of the replication fork ${ }^{120}$, where it interacts with DNA gyrase or topoisomerase IV to relieve positive superhelical stress $^{170}$. Indeed, GapR deletion is associated with a lengthened S phase and stalling of the replication fork ${ }^{120}$. Furthermore, GapR binds the parS locus, at which it plays a role in the segregation of newly replicated sister origins $^{121}$. Sister chromosome segregation in C. crescentus constitutes a ParA-independent slow step that involves the separation of the pair of parS-ParB nucleoprotein complexes and a ParA-dependent fast step that localizes one of the sister origins to the opposite pole. GapR regulates the initial slow step of segregation, as evidenced by the remerging of resolved parS-ParB complexes in $\triangle$ gapR cells ${ }^{121}$.

The NAP SlmA also controls cell cycle progres$\operatorname{sion}^{171}$. SlmA bound to SlmA-binding sites (SBSs) on the chromosome is involved in signalling the polymerization of the cytokinetic FtsZ ring ${ }^{115}$. As a nucleoid occlusion factor, SImA also ensures that the FtsZ ring is precisely positioned around the site of Ter decatenation to prevent the 'guillotining' of the chromosome ${ }^{172}$. 
A

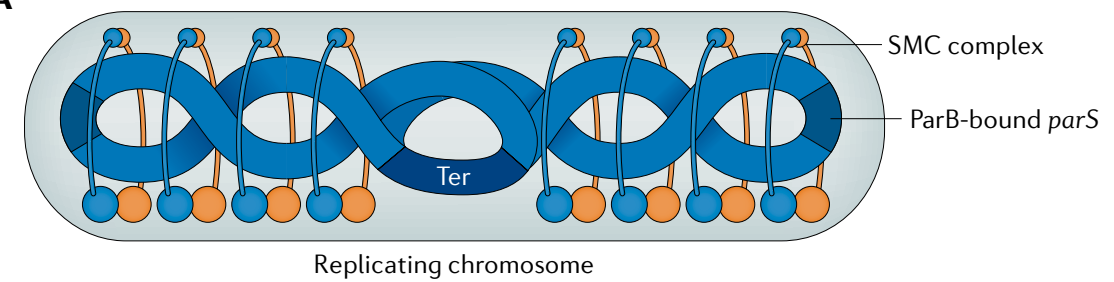

Ba

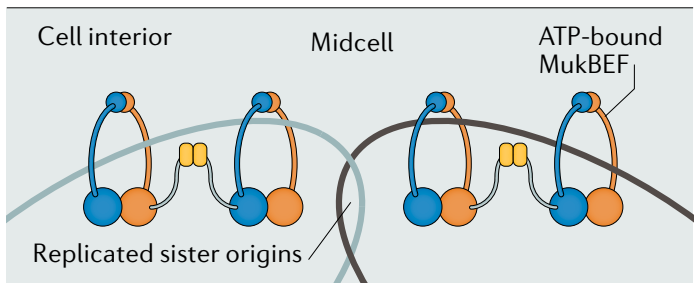

ATP-bound MukBEF complexes are recruited to the origin of replication

Bc

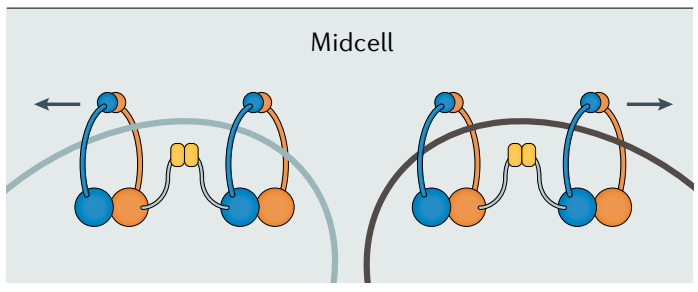

The decatenated origins, associated with MuKBEF, move away from midcell
Bb

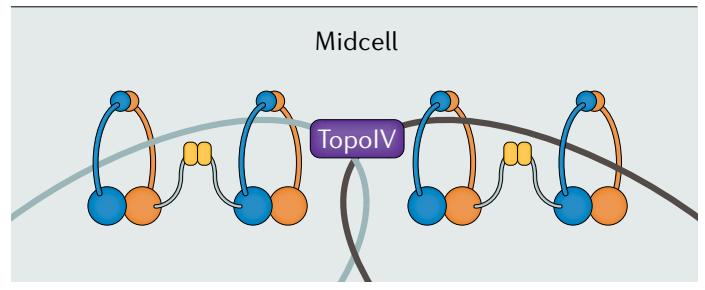

MukB recruits TopolV, which decatenates replicated sister origins

\section{Bd}

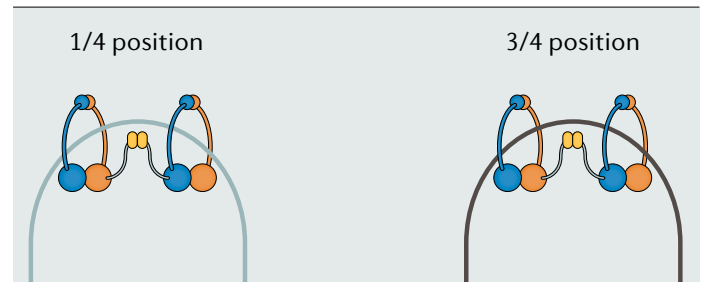

Origins localize at quarter positions in the cell

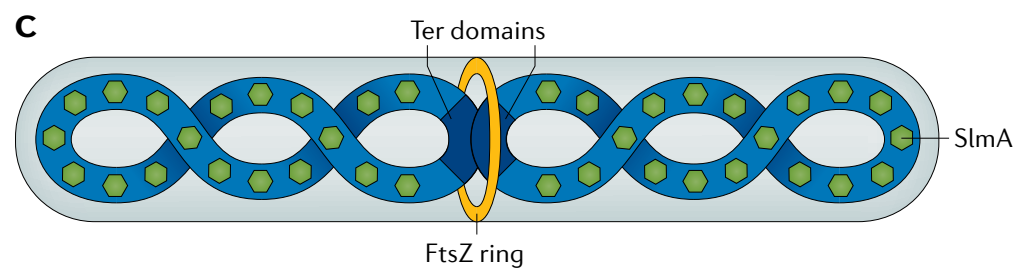

Fig. 7 | Chromosome organization has an impact on chromosome segregation and cell cycle progression.

A | ParAB-parS-mediated chromosome segregation. Structural maintenance of chromosomes (SMC) complexes are loaded at ParB-bound parS sites in the origin domain ${ }^{33,37,39}$. SMC complexes progressively move along the chromosome (not to scale) towards the terminus (Ter) domain, driving the alignment of chromosome replichores and promoting the segregation of sister chromosomes ${ }^{38,44,81}$. For ease of representation, the handcuffing model (FIC. 2, bottom) is not shown. B | MukBEF-mediated origin segregation. Ba | ATP-bound MukBEF complexes are recruited to the replicated origins ${ }^{34,163,164}$. Bb | MukB recruits topoisomerase IV (TopolV), a type II topoisomerase, which decatenates the entangled sister origins ${ }^{165,166}$. $\mathbf{B c}, \mathbf{B d} \mid$ Once decatenated, the sister origins, associated with MukBEF, move from the midcell position towards the quarter positions in the cell ${ }^{34,105}$. The segregation is proposed to be driven by a self-organizing gradient of MukBEF and the origin of replication ${ }^{167}$. C | Cell cycle progression. Cell division requires FtsZ ring assembly. FtsZ polymerization occurs at midcell, where the segregating Ter domains are located and SImA is occluded. DNA-bound SImA promotes the depolymerization of FtsZ at non-Ter regions, preventing 'guillotining' of the chromosome ${ }^{115,171-173}$.

SlmA plays its role by regulating the dynamics of FtsZ polymerization within phase-separated FtsZ droplets. In membrane-bound phase-separated systems, FtsZ polymerizes within phase-separated droplets to form filaments at membrane boundaries. The presence of SBS-bound SImA counteracts this polymerization ${ }^{171}$. This suggests that in FtsZ phase-separated structures in the bacterial cytoplasm, SBS-bound SImA antagonizes the assembly of the FtsZ ring $^{171}$. In E. coli, SBSs occur throughout the chromosome except at the Ter region ${ }^{115,173}$. This way, an SlmA-free region is produced within the cell when replication reaches the chromosome terminus. The FtsZ ring assembles at this site to initiate bacterial cytokinesis ${ }^{171}$ (FIG. 7C). The precise positioning of the FtsZ ring is reinforced by MatP-mediated condensation of the Ter macrodomain ${ }^{11}$. MatP also interacts with the septal proteins ZapA and ZapB to position Ter at midcell ${ }^{114}$. 


\section{Conclusions and future perspectives}

The past 10 years has seen the establishment of broadly applicable models for the folding of bacterial chromosomes. DNA-bending and DNA-bridging proteins play a key role in chromosome folding at the level of individual genes and in the formation of CIDs with sizes up to $300 \mathrm{~kb}$. Higher-order chromosome folding leads to the formation of macrodomains. Although these principles have been best studied in E. coli, biased binding of proteins across the chromosomes of distantly related bacteria suggests widespread relevance ${ }^{119-121}$. Future challenges include better understanding local changes in DNA folding and how these impact on other nucleic acid transactions within living cells. For example, biophysical techniques have defined the structures that H-NS can form with nucleic acids in vitro but it is still not clear whether or how such structures impact transcription in vivo. Furthermore, although we understand how individual NAPs organize DNA, it is not obvious how the concerted efforts of all NAPs combine within cells. In that light, it is of particular interest that many non-model organisms seem to harbour NAPs with enhanced functionality, for instance combining the ability to bend and bridge in a single protein ${ }^{174}$. While it is expected that general principles of DNA organization are conserved throughout the bacterial domain of life, and in fact all domains of life ${ }^{175}$, we speculate that bacteria occupying extreme environmental niches may have fine-tuned the molecular mechanisms to better cope with environmental challenges.

Published online 25 November 2019
1. Cairns, J. in Cold Spring Harbor Symposia on Quantitative Biology Vol. 28 43-46 (1963).

2. Olins, D. E. \& Olins, A. L. Physical studies of isolated eucaryotic nuclei. J. Cell Biol. 53, 715-736 (1972). Baker, J. R. The cell-theory: a restatement, history and critique. Q. J. Microsc. Sci. 96, 449-481 (1955).

4. Dame, R. T., Kalmykowa, O. J. \& Grainger, D. C. Chromosomal macrodomains and associated proteins: implications for DNA organization and replication in gram negative bacteria. PLOS Genet. 7, e1002123 (2011).

5. Grainger, D. C. Structure and function of bacterial H-NS protein. Biochem. Soc. Trans. 44, 1561-1569 (2016)

6. Gordon, B. R. et al. Structural basis for recognition of AT-rich DNA by unrelated xenogeneic silencing proteins. Proc. Natl Acad. Sci. USA 108, 10690-10695 (2011)

A molecular explanation for the recognition of AT-rich DNA by H-NS

7. Grainger, D. C., Hurd, D., Goldberg, M. D. \& Busby, S. J. Association of nucleoid proteins with coding and non-coding segments of the Escherichia coli genome. Nucleic Acids Res. 34, 4642-4652 (2006).

8. Oshima, T., Ishikawa, S., Kurokawa, K., Aiba, H. \& Ogasawara, N. Escherichia coli histone-like protein $\mathrm{H}-\mathrm{NS}$ preferentially binds to horizontally acquired DNA in association with RNA polymerase. DNA Res. 13, 141-153 (2006)

9. Navarre, W. W. et al. Selective silencing of foreign DNA with low CC content by the H-NS protein in Salmonella. Science 313, 236-238 (2006). The first description of global DNA binding by a NAP and demonstration of the role H-NS plays in silencing foreign DNA. Concurrently, similar findings were reported by Grainger et al. (2006), Oshima et al. (2006) and Lucchini et al. (2006).

10. Lucchini, S. et al. H-NS mediates the silencing of laterally acquired genes in bacteria. PLOS Pathog. 2 e81 (2006).

11. Kahramanoglou, C. et al. Direct and indirect effects of $\mathrm{H}-\mathrm{NS}$ and Fis on global gene expression control in Escherichia coli. Nucleic Acids Res. 39, 2073-2091 (2011).

12. Arold, S. T., Leonard, P. G., Parkinson, G. N. \& Ladbury, J. E. H-NS forms a superhelical protein scaffold for DNA condensation. Proc. Natl Acad. Sci. USA 107, 15728-15732 (2010). Structural biology is used to demonstrate how H-NS can polymerize by sequential head-to-head and tail-to-tail contacts.

13. Dame, R. T., Noom, M. C. \& Wuite, G. J. Bacterial chromatin organization by H-NS protein unravelled using dual DNA manipulation. Nature 444, 387-390 (2006).

14. Dame, R. T., Wyman, C. \& Goosen, N. H-NS mediated compaction of DNA visualised by atomic force microscopy. Nucleic Acids Res. 28, 3504-3510 (2000).

15. Gordon, B. R. et al. Lsr2 is a nucleoid-associated protein that targets AT-rich sequences and virulence genes in mycobacterium tuberculosis. Proc. Natl Acad. Sci. USA 107, 5154-5159 (2010)

16. Smits, W. K. \& Grossman, A. D. The transcriptional regulator Rok binds A+T-rich DNA and is involved in repression of a mobile genetic element in bacillus subtilis. PLOS Genet. 6, e1001207 (2010)

17. Duan, B. et al. How bacterial xenogeneic silencer rok distinguishes foreign from self DNA in its resident genome. Nucleic Acids Res 46, 10514-10529 (2018).

18. Ding, P. et al. A novel AT-rich DNA recognition mechanism for bacterial xenogeneic silencer MvaT. PLOS Pathog. 11, e 1004967 (2015).

19. Nolivos, S. \& Sherratt, D. The bacterial chromosome: architecture and action of bacterial SMC and SMC-like complexes. FEMS Microbiol. Rev. 38, 380-392 (2014).

20. Palecek, J. J. \& Gruber, S. Kite proteins: a superfamily of SMC/kleisin partners conserved across bacteria, archaea, and eukaryotes. Structure 23, 2183-2190 (2015).

21. Schleiffer, A. et al. Kleisins: a superfamily of bacterial and eukaryotic SMC protein partners. Mol. Cell. 11 571-575 (2003)

22. Wells, J. N., Gligoris, T. G., Nasmyth, K. A. \& Marsh, J. A. Evolution of condensin and cohesin complexes driven by replacement of kite by hawk proteins. Curr. Biol. 27, R17-R18 (2017).

23. Melby, T. E., Ciampaglio, C. N., Briscoe, G. \& Erickson, H. P. The symmetrical structure of structural maintenance of chromosomes (SMC) and MukB proteins: long, antiparallel coiled coils, folded at a flexible hinge. J. Cell Biol. 142, 1595-1604 (1998).

24. Cuylen, S., Metz, J. \& Haering, C. H. Condensin structures chromosomal DNA through topological links. Nat. Struct. Mol. Biol. 18, 894-901 (2011)

25. Gruber, S., Haering, C. H. \& Nasmyth, K. Chromosomal cohesin forms a ring. Cell 112, 765-777 (2003).

26. Zhang, N. et al. A handcuff model for the cohesin complex. J. Cell Biol. 183, 1019-1031 (2008)

27. Minnen, A Attaiech, L., Thon, M., Gruber S. $\&$ Veening, J. W. SMC is recruited to oriC by ParB and promotes chromosome segregation in streptococcus pneumoniae. Mol. Microbiol. 81, 676-688 (2011)

28. Schwartz, M. A. \& Shapiro, L. An S. M. C. ATPase mutant disrupts chromosome segregation in Caulobacter. Mol. Microbiol. 82, 1359-1374 (2011).

29. Hiraga, S. et al. Chromosome partitioning in Escherichia coli: novel mutants producing anucleate cells J Bacteriol. 171, 1496-1505 (1989).

30. Niki, H., Jaffe, A., Imamura, R., Ogura, T. \& Hiraga, S The new gene mukB codes for a 177 kd protein with coiled-coil domains involved in chromosome partitioning of E. coli. EMBO J. 10, 183-193 (1991).

31. Jensen, R. B. \& Shapiro, L. The Caulobacter crescentus smc gene is required for cell cycle progression and chromosome segregation. Proc. Natl Acad. Sci. USA 96, 10661-10666 (1999).

32. Moriya, S. et al. A Bacillus subtilis gene-encoding protein homologous to eukaryotic SMC motor protein is necessary for chromosome partition. Mol. Microbiol. 29, 179-187 (1998)

33. Wang, X., Tang, O. W., Riley, E. P. \& Rudner, D. Z. The SMC condensin complex is required for origin segregation in Bacillus subtilis. Curr. Biol. 24 287-292 (2014).

34. Danilova, O., Reyes-Lamothe, R., Pinskaya, M., Sherratt, D. \& Possoz, C. MukB colocalizes with the oriC region and is required for organization of the two
Escherichia coli chromosome arms into separate cell halves. Mol. Microbiol. 65, 1485-1492 (2007).

35. Petrushenko, Z. M., She, W. \& Rybenkov, V. V. A new family of bacterial condensins. Mol. Microbiol. 81, 881-896 (2011)

36. Yu, W., Herbert, S., Graumann, P. L. \& Gotz, F. Contribution of SMC (structural maintenance of chromosomes) and SpollIE to chromosome segregation in Staphylococci. J. Bacteriol. 192, 4067-4073 (2010).

37. Sullivan, N. L., Marquis, K. A. \& Rudner, D. Z Recruitment of SMC by ParB-parS organizes the origin region and promotes efficient chromosome segregation. Cell 137, 697-707 (2009).

38. Tran, N. T., Laub, M. T. \& Le, T. B. K. SMC progressively aligns chromosomal arms in Caulobacter crescentus but is antagonized by convergent transcription. Cell Rep. 20, 2057-2071 (2017).

39. Gruber, S. \& Errington, J. Recruitment of condensin to replication origin regions by $\mathrm{ParB} / \mathrm{SpoOJ}$ promotes chromosome segregation in B. subtilis. Cell 137 , 685-696 (2009).

40. Ganji, M. et al. Real-time imaging of DNA loop extrusion by condensin. Science 360, 102-105 (2018).

A direct visualization of DNA loop extrusion by an SMC protein.

41. Kim, E., Kerssemakers, J., Shaltiel, I. A., Haering, C. H. \& Dekker, C. DNA-loop extruding condensin complexes can traverse one another. Preprint at bioRxiv https://doi.org/10.1101/682864 (2019).

42. Nasmyth, K. Disseminating the genome: joining resolving, and separating sister chromatids during mitosis and meiosis. Annu. Rev. Genet. 35, 673-745 (2001).

43. Alipour, E. \& Marko, J. F. Self-organization of domain structures by DNA-loop-extruding enzymes. Nucleic Acids Res. 40, 11202-11212 (2012).

44. Wang, X., Brandao, H. B., Le, T. B., Laub, M. T. \& Rudner, D. Z. Bacillus subtilis SMC complexes juxtapose chromosome arms as they travel from origin to terminus. Science 355, 524-527 (2017).

45. Hirano, T. The ABCs of SMC proteins: two-armed ATPases for chromosome condensation, cohesion, and repair. Genes Dev. 16, 399-414 (2002).

46. Cairns, J. The bacterial chromosome and its manner of replication as seen by autoradiography. J. Mol. Biol. 6, 208-213 (1963).

47. Stella, S., Cascio, D. \& Johnson, R. C. The shape of the DNA minor groove directs binding by the DNAbending protein Fis. Genes Dev. 24, 814-826 (2010).

48. Hancock, S. P. Stella, S. Cascio, D. \& Johnson, R. C. DNA sequence determinants controlling affinity, stability and shape of DNA complexes bound by the nucleoid protein Fis. PLOS ONE 11, e0150189 (2016).

49. Schneider, R et al. An architectural role of the Escherichia coli chromatin protein FIS in organizing DNA. Nucleic Acids Res. 29, 5107-5114 (2001).

50. Cosgriff, S. et al. Dimerization and DNA-dependent aggregation of the Escherichia coli nucleoid protein and chaperone CbpA. Mol. Microbiol. 77 1289-1300 (2010).

51. Rice, P. A., Yang, S., Mizuuchi, K. \& Nash, H. A. Crystal structure of an IHF-DNA complex: a protein-induced DNA U-turn. Cell 87, 1295-1306 (1996). 
52. Hales, L. M., Gumport, R. I. \& Gardner, J. F. Determining the DNA sequence elements required for binding integration host factor to two different target sites. J. Bacteriol. 176, 2999-3006 (1994).

53. Rouviere-Yaniv, J. \& Gros, F. Characterization of a novel, low-molecular-weight DNA-binding protein from Escherichia coli. Proc. Natl Acad. Sci. USA 72. 3428-3432 (1975)

54. Grove, A. Functional evolution of bacterial histone-like HU proteins. Curr. Issues Mol. Biol. 13, 1-12 (2011).

55. Swinger, K. K., Lemberg, K. M., Zhang, Y. \& Rice, P. A Flexible DNA bending in HU-DNA cocrystal structures. EMBO J. 22, 3749-3760 (2003)

56. van Noort, J., Verbrugge, S., Goosen, N., Dekker, C. \& Dame, R. T. Dual architectural roles of HU: formation of flexible hinges and rigid filaments. Proc. Natl Acad. Sci. USA 101, 6969-6974 (2004).

57. Swinger, K. K. \& Rice, P. A. Structure-based analysis of HU-DNA binding. J. Mol. Biol. 365, 1005-1016 (2007).

58. Swinger, K. K. \& Rice, P. A. IHF and HU: flexible architects of bent DNA. Curr. Opin. Struct. Biol. 14, 28-35 (2004).

59. Bensaid, A., Almeida, A., Drlica, K. \& Rouviere-Yaniv, J. Crosstalk between topoisomerase I and $\mathrm{HU}$ in Escherichia coli. J. Mol. Biol. 256, 292-300 (1996).

60. Ghosh, S., Mallick, B. \& Nagaraja, V. Direct regulation of topoisomerase activity by a nucleoid-associated protein. Nucleic Acids Res. 42, 11156-11165 (2014)

61. Guo, F. \& Adhya, S. Spiral structure of Escherichia coli HUalphabeta provides foundation for DNA supercoiling. Proc. Natl Acad. Sci. USA 104 4309-4314 (2007)

62. Witz, G. \& Stasiak, A. DNA supercoiling and its role in DNA decatenation and unknotting. Nucleic Acids Res. 38, 2119-2133 (2010).

63. van der Valk, R. A. et al. Mechanism of environmentally driven conformational changes that modulate H-NS DNA-bridging activity. eLife 6, e27369 (2017). A molecular description of the function of $\mathrm{H}-\mathrm{NS}$ as an environmental sensor.

64. Boudreau, B. A. et al. StpA and Hha stimulate pausing by RNA polymerase by promoting DNA-DNA bridging of H-NS filaments. Nucleic Acids Res. 46, 5525-5546 (2018).

65. Johansson, J. \& Uhlin, B. E. Differential proteasemediated turnover of $\mathrm{H}-\mathrm{NS}$ and StpA revealed by a mutation altering protein stability and stationaryphase survival of Escherichia coli. Proc. Natl Acad. Sci. USA 96, 10776-10781 (1999)

66. Johansson, J., Eriksson, S., Sonden, B., Wai, S. N. \& Uhlin, B. E. Heteromeric interactions among nucleoidassociated bacterial proteins: localization of StpAstabilizing regions in $\mathrm{H}-\mathrm{NS}$ of Escherichia coli. J. Bacteriol. 183, 2343-2347 (2001)

67. Uyar, E. et al. Differential binding profiles of StpA in wild type and h-ns mutant cells: a comparative analysis of cooperative partners by chromatin immunoprecipitation-microarray analysis. J. Bacteriol. 191, 2388-2391 (2009).

68. Madrid, C., Balsalobre, C., Garcia, J. \& Juarez, A. The novel Hha/YmoA family of nucleoid-associated proteins: use of structural mimicry to modulate the activity of the H-NS family of proteins. Mol. Microbiol. 63, 7-14 (2007)

69. Ali, S. S. et al. Structural insights into the regulation of foreign genes in salmonella by the Hha/H-NS complex. J. Biol. Chem. 288, 13356-13369 (2013).

70. Aravind, L. \& Landsman, D. AT-hook motifs identified in a wide variety of DNA-binding proteins. Nucleic Acids Res. 26, 4413-4421 (1998)

71. Galande, S., Purbey, P. K., Notani, D. \& Kumar, P. P. The third dimension of gene regulation: organization of dynamic chromatin loopscape by SATB1. Curr. Opin. Genet. Dev. 17, 408-414 (2007).

72. Naik, R. \& Galande, S. SATB family chromatin organizers as master regulators of tumour progression. Oncogene 38, 1989-2004 (2019).

73. Yasui, D., Miyano, M., Cai, S., Varga-Weisz, P. \& Kohwi-Shigematsu, T. SATB 1 targets chromatin remodelling to regulate genes over long distances. Nature 419, 641-645 (2002)

74. Mattiroli, F. et al. Structure of histone-based chromatin in Archaea. Science 357, 609-612 (2017).

75. Henneman, B., van Emmerik, C., van Ingen, $\mathrm{H}$ $\&$ Dame, R. T. Structure and function of archaeal histones. PLOS Genet. 14, e1007582 (2018).

76. Kuhn, M. L. et al. Structural, kinetic and proteomi characterization of acetyl phosphate-dependent bacterial protein acetylation. PLOS ONE 9, e94816 (2014).
77. Schmidt, A. et al. The quantitative and conditiondependent Escherichia coli proteome. Nat. Biotechnol. 34, 104-110 (2016)

78. Weinert, B. T. et al. Acetyl-phosphate is a critical determinant of lysine acetylation in E. coli. Mol. Cell 51, 265-272 (2013)

79. Dilweg I W. \& Dame, R. T. Post-translational modification of nucleoid-associated proteins: an extra layer of functional modulation in bacteria? Biochem Soc. Trans. 46, 1381-1392 (2018)

80. Le, T. B., Imakaev, M. V., Mirny, L. A. \& Laub, M. T. High-resolution mapping of the spatial organization of a bacterial chromosome. Science 342, 731-734 (2013).

The first high-resolution three-dimensional map of a folded bacterial chromosome.

81. Marbouty, M. et al. Condensin- and replication mediated bacterial chromosome folding and origin condensation revealed by $\mathrm{Hi}-\mathrm{C}$ and super-resolution imaging. Mol. Cell 59, 588-602 (2015).

82. Sexton, T et al. Three-dimensional folding and functional organization principles of the drosophila genome. Cell 148, 458-472 (2012).

83. Nora, E. P. et al. Spatial partitioning of the regulatory landscape of the X-inactivation centre. Nature $\mathbf{4 8 5}$ 381-385 (2012).

84. Dixon, J. R. et al. Topological domains in mammalian genomes identified by analysis of chromatin interactions. Nature 485, 376-380 (2012).

85. Le, T. B. $\&$ Laub, M. T. Transcription rate and transcript length drive formation of chromosomal interaction domain boundaries. EMBO J. 35, 1582-1595 (2016).

A demonstration that transcription can control CID boundaries in $C$. crescentus.

86. Lioy, V. S. et al. Multiscale structuring of the E. coli chromosome by nucleoid-associated and condensin proteins. Cell 172, 771-783.e18 (2018).

87. Woldringh, C. L. The role of co-transcriptional translation and protein translocation (transertion) in bacterial chromosome segregation. Mol. Microbiol. 45, 17-29 (2002)

88. Fudenberg, G. et al. Formation of chromosoma domains by loop extrusion. Cell Rep. 15, 2038-2049 (2016).

89. Sanborn, A. L. et al. Chromatin extrusion explains key features of loop and domain formation in wild type and engineered genomes. Proc. Natl Acad. Sci. USA 112, E6456-E6465 (2015)

90. Rao, S. S. et al. A 3D map of the human genome at kilobase resolution reveals principles of chromatin looping. Cell 159, 1665-1680 (2014). A high-resolution interaction map of eukaryotic chromosomes

91. Rowley, M. J. et al. Evolutionarily conserved principles predict 3D chromatin organization. Mol. Cell 67 , 837-852.e7 (2017).

92. Vietri Rudan, M. et al. Comparative $\mathrm{Hi}-\mathrm{C}$ reveals that CTCF underlies evolution of chromosomal domain architecture. Cell Rep. 10, 1297-1309 (2015)

93. Cubenas-Potts, C. et al. Different enhancer classes in Drosophila bind distinct architectural proteins and mediate unique chromatin interactions and 3D architecture. Nucleic Acids Res. 45, 1714-1730 (2017).

94. Wang, Q., Sun, O., Czajkowsky, D. M. \& Shao, Z. Sub-kb Hi-C in D. melanogaster reveals conserved characteristics of TADs between insect and mammalian cells. Nat. Commun. 9, 188 (2018).

95. Ulianov, S. V. et al. Active chromatin and transcription play a key role in chromosome partitioning into topologically associating domains. Genome Res. 26 70-84 (2016)

96. Hsieh, T. H. et al. Mapping nucleosome resolution chromosome folding in yeast by Micro-C. Cell 162, 108-119 (2015).

97. Lal, A. et al. Genome scale patterns of supercoiling in a bacterial chromosome. Nat. Commun. 7, 11055 (2016).

98. Bermudez, I., Garcia-Martinez, J., Perez-Ortin, J. E. $\S$ Roca, J. A method for genome-wide analysis of DNA helical tension by means of psoralen-DNA photobinding. Nucleic Acids Res. 38, e182 (2010).

99. Ali Azam, T., Iwata, A., Nishimura, A., Ueda, S. \& Ishihama, A. Growth phase-dependent variation in protein composition of the Escherichia coli nucleoid. J. Bacteriol. 181, 6361-6370 (1999).

100. Rovinskiy, N., Agbleke, A. A., Chesnokova, O., Pang, Z. $\S$ Higgins, N. P. Rates of gyrase supercoiling and transcription elongation control supercoil density in a bacterial chromosome. PLOS Genet. 8, e 1002845 (2012).
101. Umbarger, M. A. et al. The three-dimensional architecture of a bacterial genome and its alteration by genetic perturbation. Mol. Cell 44, 252-264 (2011).

102. Uhlmann, F. SMC complexes: from DNA to chromosomes. Nat. Rev. Mol. Cell Biol. 17, 399-412 (2016).

103. Fennell-Fezzie, R., Gradia, S. D., Akey, D. \& Berger, J. M. The MukF subunit of Escherichia coli condensin: architecture and functional relationship to kleisins. EMBO J. 24, 1921-1930 (2005).

104. Woo, J. S. et al. Structural studies of a bacterial condensin complex reveal ATP-dependent disruption of intersubunit interactions. Cell 136, 85-96 (2009)

105. Badrinarayanan, A., Reyes-Lamothe, R., Uphoff, S., Leake, M. C. \& Sherratt, D. J. In vivo architecture and action of bacterial structural maintenance of chromosome proteins. Science 338, 528-531 (2012)

106. Chen, N. et al. ATP-induced shrinkage of DNA with MukB protein and the MukBEF complex of Escherichia coli. J. Bacteriol. 190, 3731-3737 (2008).

107. Zawadzka, K. et al. MukB ATPases are regulated ndependently by the $\mathrm{N}$ - and C-terminal domains of MukF kleisin. eLife 7, e31522 (2018).

108. Espeli, O., Mercier, R. \& Boccard, F. DNA dynamics vary according to macrodomain topography in the E. coli chromosome. Mol. Microbiol. 68, 1418-1427 (2008)

109. Valens, M., Thiel, A. \& Boccard, F. The MaoP/maoS site-specific system organizes the ori region of the E. coli chromosome into a macrodomain. PLOS Genet 12, e1006309 (2016).

110. Duigou, S. \& Boccard, F. Long range chromosome organization in Escherichia coli: The position of the replication origin defines the non-structured regions and the right and left macrodomains. PLOS Genet. 13, e1006758 (2017)

111. Mercier, R. et al. The MatP/matS site-specific system organizes the terminus region of the $E$. coli chromosome into a macrodomain. Cell 135 , 475-485 (2008).

112. Dupaigne, P. et al. Molecular basis for a proteinmediated DNA-bridging mechanism that functions in condensation of the E. coli chromosome. Mol. Cell 48 560-571 (2012).

Mercier et al. (2008) and Dupaigne et al. (2012) provide the first molecular explanations for the formation of a chromosomal macrodomain in bacteria.

113. Thiel, A., Valens, M., Vallet-Gely, I., Espeli, O. \& Boccard, F. Long-range chromosome organization in E. coli: a site-specific system isolates the Ter macrodomain. PLOS Genet. 8, e1002672 (2012).

114. Espeli, O. et al. A MatP-divisome interaction coordinates chromosome segregation with cell division in E. coli. EMBO J. 31, 3198-3211 (2012).

115. Cho, H., McManus, H. R., Dove, S. L. \& Bernhardt, T. C Nucleoid occlusion factor SImA is a DNA-activated FtsZ polymerization antagonist. Proc. Natl Acad. Sci. USA 108, 3773-3778 (2011).

116. Sanchez-Romero, M. A. et al. Dynamic distribution of seqa protein across the chromosome of Escherichia coli K-12. mBio 1, e00012-10 (2010).

117. Lu, M., Campbell, J. L., Boye, E. \& Kleckner, N. SeqA: a negative modulator of replication initiation in $E$. coli. Cell 77, 413-426 (1994).

118. Slater, S. et al. E. coli SeqA protein binds oriC in two different methyl-modulated reactions appropriate to its roles in DNA replication initiation and origin sequestration. Cell 82, 927-936 (1995).

119. Wu, L. J. et al. Noc protein binds to specific DNA sequences to coordinate cell division with chromosome segregation. EMBO J. 28, 1940-1952 (2009).

120. Arias-Cartin, R. et al. Replication fork passage drives asymmetric dynamics of a critical nucleoid-associated protein in Caulobacter. EMBO J. 36, 301-318 (2017).

121. Taylor, J. A., Panis, G., Viollier, P. H. \& Marczynski, G. T A novel nucleoid-associated protein coordinates chromosome replication and chromosome partition Nucleic Acids Res. 45, 8916-8929 (2017).

122. Oin, L. et al. Structural basis for osmotic regulation of the DNA binding properties of H-NS proteins. Preprin at bioRxiv https://doi.org/10.1101/757732 (2019).

123. Ono, S. et al. H-NS is a part of a thermally controlled mechanism for bacterial gene regulation. Biochem. $J$. 391, 203-213 (2005).

124. Goransson, M. et al. Transcriptional silencing and thermoregulation of gene expression in Escherichia coli. Nature 344, 682-685 (1990). 
125. Amit, R., Oppenheim, A. B. \& Stavans, J. Increased bending rigidity of single DNA molecules by H-NS, a temperature and osmolarity sensor. Biophys J. 84 2467-2473 (2003)

126. Kotlajich, M. V. et al. Bridged filaments of histone-like nucleoid structuring protein pause RNA polymerase and aid termination in bacteria. eLife 4, e04970 (2015). Highlights the role of DNA architecture in transcriptional regulation

127. Meyer, A. S. \& Grainger, D. C. The Escherichia coli nucleoid in stationary phase. Adv. Appl. Microbiol. 83, 69-86 (2013).

128. Ball, C. A., Osuna, R., Ferguson, K. C. \& Johnson, R. C. Dramatic changes in Fis levels upon nutrient upshift in Escherichia coli. J. Bacteriol. 174, 8043-8056 (1992).

129. Almiron, M., Link, A. J., Furlong, D. \& Kolter, R. A novel DNA-binding protein with regulatory and protective roles in starved Escherichia coli. Genes Dev. 6, 2646-2654 (1992).

130. Karas, V. O., Westerlaken, I. \& Meyer, A. S. The DNAbinding protein from starved cells (Dps) utilizes dual functions to defend cells against multiple stresses. J. Bacteriol. 197, 3206-3215 (2015)

131. Wolf, S. G. et al. DNA protection by stress-induced biocrystallization. Nature 400, 83-85 (1999). Demonstrates that the chromosome of starved E. coli cells is reorganized by Dps to form a biocrystal

132. Ohniwa, R. L. et al. Dynamic state of DNA topology is essential for genome condensation in bacteria. $E M B O$ J. 25, 5591-5602 (2006)

133. Janissen, R. et al. Global DNA compaction in stationary-phase bacteria does not affect transcription Cell 174, 1188-1199.e14 (2018). Highlights the role of phase separation in compartmentalization of the bacterial cell

134. Lamberte, L. E. et al. Horizontally acquired AT-rich genes in Escherichia coli cause toxicity by sequestering RNA polymerase. Nat. Microbiol. 2, 16249 (2017).

135. Singh, S. S. \& Grainger, D. C. H-NS can facilitate specific DNA-binding by RNA polymerase in AT-rich gene regulatory regions. PLOS Genet. 9, e1003589 (2013).

136. Singh, S. S. et al. Widespread suppression of intragenic transcription initiation by H-NS. Genes Dev. 28, 214-219 (2014). Demonstrates that H-NS plays a key role in preventing the spurious initiation of transcription.

137. Myers, K. S. et al. Genome-scale analysis of Escherichia coli FNR reveals complex features of transcription factor binding. PLOS Genet. 9, e1003565 (2013)

138. Haycocks, J. R., Sharma, P., Stringer, A. M., Wade, J. T. \& Grainger, D. C. The molecular basis for control of ETEC enterotoxin expression in response to environment and host. PLOS Pathog. 11, e1004605 (2015).

139. Shin, M. et al. DNA looping-mediated repression by histone-like protein H-NS: specific requirement of Esigma70 as a cofactor for looping. Genes Dev. 19 2388-2398 (2005)

140. Dame, R. T., Wyman, C., Wurm, R., Wagner, R. $\Sigma$ Goosen, N. Structural basis for H-NS-mediated trapping of RNA polymerase in the open initiation complex at the rrnB P1. J. Biol. Chem. 277, 2146-2150 (2002)

141. Shin, M. et al. Gene silencing by H-NS from distal DNA site. Mol. Microbiol. 86, 707-719 (2012).

142. Dattananda, C. S., Rajkumari, K. \& Gowrishankar, J. Multiple mechanisms contribute to osmotic inducibility of proU operon expression in Escherichia coli: demonstration of two osmoresponsive promoters and of a negative regulatory element within the first structural gene. J. Bacteriol. 173, 7481-7490 (1991)

143. Gowrishankar, J. Nucleotide sequence of the osmoregulatory proU operon of Escherichia coli. J. Bacteriol 171, 1923-1931 (1989).

144. Lucht, J. M., Dersch, P., Kempf, B. \& Bremer, E. Interactions of the nucleoid-associated DNA-binding protein $\mathrm{H}-\mathrm{NS}$ with the regulatory region of the osmotically controlled proU operon of Escherichia coli. J. Biol. Chem. 269, 6578-6578 (1994)

145. Nagarajavel, V., Madhusudan, S., Dole, S., Rahmouni, A. R. \& Schnetz, K. Repression by binding of H-NS within the transcription unit. J. Biol. Chem. 282, 23622-23630 (2007)
146. Madrid, C., Nieto, J. M. \& Juarez, A. Role of the Hha/ $Y m o A$ family of proteins in the thermoregulation of the expression of virulence factors. Int. J. Med. Microbiol. 291, 425-432 (2002)

147. Wade, J. T. \& Grainger, D. C. Waking the neighbours: disruption of H-NS repression by overlapping transcription. Mol Microbiol 108, 221-225 (2018).

148. Mai, X., Chou, S. \& Struhl, K. Preferential accessibility of the yeast his3 promoter is determined by a general property of the DNA sequence, not by specific elements. Mol. Cell Biol. 20, 6668-6676 (2000)

149. Schneider, D. A., Ross, W. \& Gourse, R. L. Control of rRNA expression in Escherichia coli. Curr. Opin. Microbiol. 6, 151-156 (2003)

150. Browning, D. F., Cole, J. A. \& Busby, S. J. Suppression of FNR-dependent transcription activation at the Escherichia coli nir promoter by Fis, IHF and $\mathrm{H}-\mathrm{NS}$ : modulation of transcription initiation by a complex nucleo-protein assembly. Mol. Microbiol. 37 , 1258-1269 (2000).

151. Browning, D. F. et al. Integration of three signals at the Escherichia coli nrf promoter: a role for Fis protein in catabolite repression. Mol. Microbiol. 57, 496-510 (2005).

152. Bokal, A. J., Ross, W., Gaal, T., Johnson, R. C. \& Gourse, R. L. Molecular anatomy of a transcription activation patch: FIS-RNA polymerase interactions at the Escherichia coli rrnB P1 promoter. EMBO J. 16, 154-162 (1997)

153. McLeod, S. M., Aiyar, S. E., Gourse, R. L. \& Johnson, R. C. The C-terminal domains of the RNA polymerase alpha subunits: contact site with Fis and localization during co-activation with CRP at the Escherichia coli proP P2 promoter. J. Mol. Biol. 316 517-529 (2002)

154. Semsey, S., Tolstorukov, M. Y., Virnik, K., Zhurkin, V. B. ¿ Adhya, S. DNA trajectory in the Gal repressosome. Genes Dev. 18, 1898-1907 (2004)

155. Oberto, J., Nabti, S., Jooste, V., Mignot, H. \& Rouviere-Yaniv, J. The HU regulon is composed of genes responding to anaerobiosis, acid stress, high osmolarity and SOS induction. PLOS ONE 4, e4367 (2009).

156. Mangan, M. W. et al. Nucleoid-associated protein $\mathrm{HU}$ controls three regulons that coordinate virulence, response to stress and general physiology in Salmonella enterica serovar Typhimurium. Microbiology 157, 1075-1087 (2011)

157. Zhang, N., Darbari, V. C., Glyde, R., Zhang, X. \& Buck, $M$. The bacterial enhancer-dependent RNA polymerase. Biochem. J. 473, 3741-3753 (2016).

158. Browning, D. F., Beatty, C. M., Wolfe, A. J., Cole, J. A. \& Busby, S. J. Independent regulation of the divergent Escherichia coli nrfA and acsP1 promoters by a nucleoprotein assembly at a shared regulatory region. Mol. Microbiol. 43, 687-701 (2002)

159. Toro, E \& Shapiro, L. Bacterial chromosome organization and segregation. Cold Spring Harb. Perspect. Biol. 2, a000349 (2010).

160. Wang, X., Montero Llopis, P. \& Rudner, D. Z. Organization and segregation of bacterial chromosomes. Nat. Rev. Genet. 14, 191-203 (2013).

161. Mierzejewska, J. \& Jagura-Burdzy, G. Prokaryotic ParA-ParB-parS system links bacterial chromosome segregation with the cell cycle. Plasmid 67, 1-14 (2012).

162. Toro, E., Hong, S. H., McAdams, H. H. \& Shapiro, L. Caulobacter requires a dedicated mechanism to initiate chromosome segregation. Proc. Natl Acad. Sci. USA 105, 15435-15440 (2008).

163. Adachi, S., Kohiyama, M., Onogi, T. \& Hiraga, S. Localization of replication forks in wild type and mukB mutant cells of Escherichia coli. Mol. Genet. Genomics 274, 264-271 (2005).

164. Badrinarayanan, A., Lesterlin, C., Reyes-Lamothe, R. \& Sherratt, D. The Escherichia coli SMC complex, MukBEF, shapes nucleoid organization independently of DNA replication. J. Bacteriol. 194, 4669-4676 (2012).

165. Nicolas, E. et al. The SMC complex MukBEF recruit topoisomerase IV to the origin of replication region in live Escherichia coli. mBio 5, e01001-e01013 (2014).
166. Hayama, R. \& Marians, K. J. Physical and functional interaction between the condensin MukB and the decatenase topoisomerase IV in Escherichia coli. Proc Natl Acad. Sci. USA 107, 18826-18831 (2010).

167. Hofmann, A., Makela, J., Sherratt, D. J., Heermann, D. \& Murray, S. M. Self-organized segregation of bacterial chromosomal origins. eLife 8, e46564 (2019).

168. Nolivos, S. et al. MatP regulates the coordinated action of topoisomerase IV and MukBEF in chromosome segregation. Nat. Commun. 7, 10466 (2016).

169. Ricci, D. P. et al. Cell cycle progression in caulobacter requires a nucleoid-associated protein with high AT sequence recognition. Proc. Natl Acad. Sci. USA 113, E5952-E5961 (2016)

170. Guo, M. S., Haakonsen, D. L., Zeng, W., Schumacher, M. A. \& Laub, M. T. A bacterial chromosome structuring protein binds overtwisted DNA to stimulate type II topoisomerases and enable DNA replication. Cell 175, 583-597. e523 (2018).

171. Monterroso, B. et al. Bacterial FtsZ protein forms phase-separated condensates with its nucleoidassociated inhibitor SImA. EMBO Rep. 20, e45946 (2019).

172. Bernhardt, T. G. \& de Boer, P. A. SImA, a nucleoidassociated, FtsZ binding protein required for blocking septal ring assembly over chromosomes in $E$. coli. Mol. Cell 18, 555-564 (2005).

173. Tonthat, N. K. et al. Molecular mechanism by which the nucleoid occlusion factor, SImA, keeps cytokinesis in check. EMBO J. 30, 154-164 (2011)

174. Anand, C., Garg, R., Ghosh, S. \& Nagaraja, V. A Sir2 family protein Rv1151c deacetylates $\mathrm{HU}$ to alter its DNA binding mode in Mycobacterium tuberculosis. Biochem. Biophys. Res. Commun. 493, 1204-1209 (2017).

175. Luijsterburg, M. S., White, M. F., van Driel, R. \& Dame, R. T. The major architects of chromatin: architectural proteins in bacteria, archaea and eukaryotes. Crit. Rev. Biochem. Mol. Biol. 43, 393-418 (2008)

176. Dekker, J., Rippe, K., Dekker, M. \& Kleckner, N. Capturing chromosome conformation. Science 295 1306-1311 (2002).

177. Lieberman-Aiden, E. et al. Comprehensive mapping of long-range interactions reveals folding principles of the human genome. Science 326, 289-293 (2009).

178. van Berkum, N. L. et al. Hi-C: a method to study the three-dimensional architecture of genomes. J. Vis. Exp. https://doi.org/10.3791/1869 (2010).

179. Nagano, T. et al. Single-cell Hi-C reveals cell-to-cell variability in chromosome structure. Nature 502 , 59-64 (2013).

180. Nagano, T. et al. Cell-cycle dynamics of chromosomal organization at single-cell resolution. Nature $\mathbf{5 4 7}$ 61-67 (2017)

181. Stevens, T. J. et al. 3D structures of individual mammalian genomes studied by single-cell $\mathrm{Hi}-\mathrm{C}$. Nature 544, 59-64 (2017)

Nagano et al. (2013), Nagano et al. (2017) and Stevens et al. (2017) demonstrate single-cell Hi-C in eukaryotes.

\section{Acknowledgements}

The authors thank the Netherlands Organization for Scientific Research (VICI 016.160.613) (R.T.D.), the Wellcome Trust (212193/Z/18/Z) (D.C.G.), the Leverhulme Trust (RPG-2018198) (D.C.G.), the UK Biotechnology and Biological Sciences Research Council (BB/H010289/1) (D. C. G) and the Human Frontier Science Program (HFSP; RGP0014/2014) (R.T.D. and D.C.G.) for funding of current research in their laboratories.

\section{Author contributions}

All authors researched data for the article, substantially contributed to discussion of the content and wrote the article. R.T.D. and D.C.G. reviewed and edited the manuscript before submission.

\section{Competing interests}

The authors declare no competing interests.

Publisher's note

Springer Nature remains neutral with regard to jurisdictional claims in published maps and institutional affiliations. 